\title{
FINITE GROUPS WHICH ADMIT AN AUTOMORPHISM WITH FEW ORBITS
}

\author{
DANIEL GORENSTEIN
}

1. Introduction. In the course of investigating the structure of finite groups which have a representation in the form $A B A$, for suitable subgroups $A$ and $B$, we have been forced to study groups $G$ which admit an automorphism $\phi$ such that every element of $G$ lies in at least one of the orbits under $\phi$ of the elements $g, g \phi^{\tau}(g), g \phi^{\tau}(g) \phi^{2 \tau}(g), g \phi^{\tau}(g) \phi^{2 r}(g) \phi^{3 r}(g)$, etc., where $g$ is a fixed element of $G$ and $r$ is a fixed integer.

In a previous paper on $A B A$-groups written jointly with I. N. Herstein (4), we have treated the special case $r=0$ (in which case every element of $G$ can be expressed in the form $\left.\phi^{i}\left(g^{j}\right)\right)$, and have shown that if the orders of $\phi$ and $g$ are relatively prime, then $G$ is either Abelian or the direct product of an Abelian group of odd order and the quaternion group of order 8 . In another paper (3), the author has shown that if each element of $G$ lies in exactly one of these orbits, then $G$ must be an elementary Abelian group of type $(p, p, \ldots, p)$. The purpose of this paper is to prove more generally that any finite group $G$ which admits an automorphism whose orbits are of the above form is necessarily solvable (Theorem 5 ). The burden of the proof rests on the case in which $\phi$ leaves only the identity element of $G$ fixed, and in this case we shall show that $G$ is in fact nilpotent (Theorem 4).

In the course of the proof we first establish the nilpotency of $G$ in the so-called non-exceptional case (in particular, if $G$ is solvable) (Theorem 1). For this case our statement and argument resemble a result of Feit (2) and Higman (5), which asserts that a solvable group having an automorphism of prime order which leaves only the identity element fixed is necessarily nilpotent.* Their argument actually applies if $G$ is assumed to be $p$-normal for all $p \mid o(G)$. Recently it has been announced by J. G. Thompson that $G$ must in fact be $p$-normal for all $p \mid o(G)$ whenever $G$ admits an automorphism of prime order leaving only the identity element of $G$ fixed, from which it follows that an arbitrary group $G$ admitting such an automorphism is necessarily nilpotent. $\dagger$

However, not much is known concerning the structure of $G$ if $\phi$ is of composite order. It is not difficult to construct a solvable non-nilpotent group $G$ admitting an automorphism $\phi$ of composite order leaving only the identity

\footnotetext{
Received October 22, 1958.

${ }^{*}$ Feit proves the nilpotency of $G$ under the weaker hypothesis that no subgroup of $G$ has an exceptional group as a composition factor.

†A.M.S. Notices, 5 (6) (November, 1958), 695.
} 
element of $G$ fixed; and it is an open question whether $G$ must be solvable to admit such an automorphism even when $\phi$ has order 4 .

We see then that our assumption on the orbits of $G$ is a strong one since no other conditions on $G$ or the order of $\phi$ are needed to prove that $G$ is nilpotent if $\phi$ leaves only the identity element of $G$ fixed. A direct consequence of this assumption is a simple inequality (Lemma 2.3) which exists between the order of $\phi$ and the order of $G$; and it is this inequality which lies at the heart of many of our arguments.

In $\$ \S 10$ and 11 we shall determine the structure of groups of prime power order which admit an automorphism $\phi$ without non-trivial fixed elements satisfying our special condition on orbits, and shall show that such a group is either Abelian or of class 2 (Theorem 8). Combining this result with Theorem 4 , it will follow that any group $G$ admitting such an automorphism $\phi$ without non-trivial fixed elements is either Abelian or nilpotent of class 2 (Theorem 9).

In the final section we shall determine the precise connection between groups whose orbits satisfy this condition and groups of the form $A B A$. As an application we shall prove the solvability of a certain class of $A B A$-groups (Theorem 10).

The author wishes to thank Prof. Herstein for his considerable help in the preparation of this paper, particularly with the proof of Lemma 3.1.

2. $\phi$-groups. We shall call a group $G$ a $\phi$-group if $G$ admits an automorphism $\phi$ such that every element of $G$ can be expressed in the form $\phi^{i}\left(g \phi^{r}(g) \ldots\right.$ $\left.\phi^{r(j-1)}(g)\right)$ for some fixed integer $r$ and some fixed element $g$ in $G, i$ and $j$ being arbitrary. The element $g$ will be called a generator of $G$ under $\phi$, and $r$ will be called the index of $G$ with respect to $g$, or simply the index of $G$.

For simplicity we exclude the trivial case in which the order $h$ of $\phi$ is 1 . This implies, in particular, that $o(G)>1$. We may further assume that $r \mid h$ for otherwise set $r_{1}=(r, h)$ and define $\phi_{1}=\phi^{r / r_{1}}$. Then clearly $\phi_{1}$ has order $h, G$ is a $\phi_{1}$-group of index $r_{1}$ with respect to $g$, and we have $r_{1} \mid h$. In the special case in which $h=r$, and hence in which every element of $G$ can be expressed in the form $\phi^{i}\left(g^{j}\right)$, we shall say that $G$ is a $\phi$-group of index 0 .

We can imbed $G$ as a normal subgroup of a group $G^{*}$, which contains an element $a$ of order $h$ such that $a g a^{-1}=\phi(g)$ for all $g$ in $G$ and such that $G^{*}=G A$, where $A$ denotes the subgroup generated by $a$. If $\phi$ is of prime order and leaves only the identity element of $G$ fixed, it is easy to show that $G^{*}$ is a Frobenius group and that $G$ is the regular subgroup of $G^{*}$. By analogy with this case, we shall say, whenever $\phi$ leaves only the identity element of $G$ fixed, that $G$ is a regular $\phi$-group, and that $\phi$ is a Frobenius automorphism of $G$.

For brevity we also introduce the symbol $[g]_{r}^{j}$ for the element $g \phi^{\tau}(g) \ldots$ $\phi^{r(j-1)}(g)$. For completeness we set $[g]_{r}^{0}=1$. This symbol has several formal properties which we shall use repeatedly throughout the ensuing discussion, and which for convenience we incorporate into the following lemma: 
LEMMA 2.1. Let $\phi$ be an automorphism ofo rder $h$ of a group $G$. For any $g$ in $G$ and any integers $i, j, k, r$, we have $\left[[g]_{r}{ }^{j}\right]_{r j}{ }^{k}=[g]_{r}{ }^{j k}$ and $[g]_{r}{ }^{j+k}=[g]_{r}{ }_{r} \phi^{r j}\left([g]_{r}{ }^{k}\right)$. Furthermore, if $h \mid r,[g]_{r}^{j}=g^{j}$; while if $r \mid h$ and $\phi^{r}$ is Frobenius, $[g]_{r}{ }^{h / r}=1$.

Proof. All these relations except the last follow immediately from the definition of the symbol $[g]_{r}{ }^{j}$. On the other hand, if $\phi^{r}$ leaves only the identity element of $G$ fixed, it is easy to see that $g$ can be written in the form $x^{-1} \phi^{r}(x)$ for some $x$ in $G$. But then $[g]_{r}^{h / r}=\left(x^{-1} \phi^{r}(x)\right)\left(\phi^{r}\left(x^{-1} \phi^{r}(x)\right) \ldots \phi^{h-r}\left(x^{-1} \phi^{r}(x)\right)\right.$ $=x^{-1} \phi^{h}(x)=1$.

The following lemma shows that the property of being a $\phi$-group carries over to subgroups and factor groups of $G$.

Lemma 2.2. Let $G$ be a $\phi$-group of index $r$ with respect to the generator $g$, and let $H$ be a subgroup of $G$ invariant under $\phi$. Then $H$ is a $\phi$-group of index $r s$ with respect to the generator $[g]_{r}^{s}$ for some integer $s$. If $H$ is normal in $G$ and $\bar{G}=G / H$ then $\bar{G}$ is a $\bar{\phi}$-group of index $r$ with respect to the generator $\bar{g}$, where $\bar{\phi}, \bar{g}$ denote respectively the image of $\phi$ on $\bar{G}$ and the residue of $g$ in $\bar{G}$. Furthermore, no proper subgroup of $G$ invariant under $\phi$ contains $g$.

Proof. The last two statements of the lemma follow at once from the definition of a $\phi$-group. To prove the first assertion, let $s$ be the least positive integer such that $g_{1}=[g]_{r}{ }^{s}$ is in $H$. Since $H$ is invariant under $\phi$, every element of $G$ of the form $\phi^{i}\left(\left[g_{1}\right]_{r s}{ }^{j}\right)$ is in $H$. Conversely, if $[g]_{r}{ }^{k} \in H$, write $k=s j+t$ and use Lemma 2.1 to get

$$
[g]_{r}^{k}=[g]_{r}^{s j} \phi^{r s j}\left([g]_{r}^{t}\right)=\left[g_{1}\right]_{r s}^{j} \phi^{r s j}\left([g]_{r}^{t}\right)
$$

whence $[g]_{r}{ }^{t} \in H$. Since $s$ is the least positive integer with this property, $t=0$, and it follows that every element of $H$ is of the form $\phi^{i}\left(\left[g_{1}\right]_{r s}{ }^{j}\right)$. Thus $H$ is a $\phi$-group of index $r s$ with generator $[g]_{r} s$.

Finally, we shall establish a simple, but extremely important, relation between the order of $\phi$ and the order of $G$.

Lemma 2.3. Let $G$ be a $\phi$-group of index $r$ with respect to a generator $g$ of $G$; let $h$ be the order of $\phi$ and let $k$ be the least integer such that $[g]_{r}{ }^{k}=1$. Then $h k>o(G)$. In particular, if $\phi^{r}$ is Frobenius, $k=h / r$.

Proof. Since every element of $G$ must be in the orbit under $\phi$ of one of the $k$ elements $[g]_{r}{ }^{j}, j=1,2, \ldots, k$, since each of these orbits contains at most $h$ elements, and since the last one of them consists of only the identity element of $G$, the inequality $h k>o(G)$ is immediate.

If $\phi^{r}$ is Frobenius, Lemma 2.1 shows that $[g]_{r}{ }^{h / r}=1$. The proof of this equality shows also that for any value of $j<h / r,[g]_{r}^{j} \neq 1$. Thus $k=h / r$.

3. Automorphisms of a class of groups of order $p^{m} q^{n}$. In the next three sections we shall show that a regular $\phi$-group in which no subgroup has an exceptional group as a composition factor is nilpotent. The heart of 
the problem is to prove this result for certain $\phi$-groups of order $p^{m} q^{n} ; \S \S 3$ and 4 are devoted to this special case.

Lemma 3.1. Let $G$ be a group of order $p^{m} q^{n}$, $p$ and $q$ being primes, in which the $p$-Sylow subgroup $P$ is normal in $G$ and Abelian of type $(p, p, \ldots, p)$, while the $q$-Sylow subgroups are Abelian of type $(q, q, \ldots, q)$; and assume that the centre of $G$ is trivial. Suppose $\phi$ is an automorphism of $G$ of order $h$ such that no proper subgroup of $P$ which is invariant under $\phi$ is normal in $G$ and such that some $q$-Sylow subgroup $Q$, but no proper subgroup of $Q$, is invariant under $\phi$. Then if $d$ is the order of $\phi$ on $Q$, we have $d \mid m$ and $h \mid d\left(p^{m / d}-1\right)$.

Proof. Since $G$ has no centre, $p \neq q$ and $m, n>0$.

Since each element $y$ in $Q$ induces by conjugation an automorphism $\psi_{y}$ of $P$, there exists a group of automorphisms $A$ acting on $P$ which can be expressed in the form $\bar{Q} R$, where $\bar{Q}$ is normal in $A$ and is isomorphic to $Q$ under the correspondence $\psi_{y} \leftrightarrow y$, where $R$ is the cyclic subgroup generated by $\phi$, and where

$$
\phi^{-1} \psi_{y} \phi=\psi_{\phi(y)} \text { for all } y \text { in } Q \text {. }
$$

For all $y$ in $Q$, we have $\phi^{d}(y)=y$, and hence $\phi^{-d} \psi_{y} \phi^{d}=\psi_{y}$. Thus $\phi^{d}$ is in the centre of $A$, and since $Q$ is Abelian, the subgroup $A_{0}$ generated by $\phi^{d}$ and $\bar{Q}$ is Abelian.

We shall regard $P$ as an $m$-dimensional vector space over the prime field $K$ with $p$-elements, and $A$ as a group of linear transformations acting on $P$. If $K^{*}$ denotes the algebraic closure of $K$ and $P^{*}$, the $m$-dimensional vector space over $K^{*}$, we may also consider $A$ as a group of linear transformations on $P^{*}$.

Now let $W$ be a minimal subspace of $P$, invariant under $\phi$, and of dimension $t$, and let $f(x)$, of degree $t$ and irreducible over $K$, be the minimal polynomial of $\phi^{d}$ on $W$. Since $\phi^{d}$ is in the centre of $A$, the subspaces $\phi^{i} \psi_{y}(W)$ are invariant under $\phi^{d}$ for all $i$ and all $y$ in $Q$, and $\phi^{d}$ has the same minimal polynomial $f(x)$ on each of these subspaces. Let

$$
P_{0}=\sum_{i, y} \phi^{i} \psi_{y}(W)
$$

It follows immediately from (1) that $P_{0}$ is left fixed by every element of $A$. Regarded as a subgroup of $P, P_{0}$ is thus normal in $G$ and invariant under $\phi$, whence by our hypotheses $P_{0}=P$. Since now $P$ is the sum of minimal subspaces invariant under $\phi^{d}$, it follows that $P$ is the direct sum of subspaces $W_{1}, W_{2}, \ldots, W_{s}$, each of dimension $t$, each invariant under $\phi^{d}$, and on each of which the minimal polynomial of $\phi^{d}$ is $f(x)$. Thus

$$
m=s t \text { and } f(x)^{s} \text { is the characteristic polynomial of } \phi^{d} \text { on } P \text {. }
$$

The order $w$ of $\phi^{d}$ on $P$ is the same as its order on each of the subspaces $W_{i}$, and since $f(x)$ is irreducible, it follows that $w \mid p^{t}-1$. In particular, this 
implies $(w, p)=1$, and hence that the order of $A_{0}$ is relatively prime to $p$. It follows that the representation of $A_{0}$ in $P^{*}$ is completely reducible.

Now $A_{0}$ is Abelian and $P^{*}$ has coefficients in an algebraically closed field; hence we can find a vector $x_{1} \neq 0$ in $P^{*}$ which is a common characteristic vector of every element in $A_{0}$. We shall show that for $0 \leqslant i<d$ the vectors $\phi^{i}\left(x_{1}\right)$ are also common characteristic vectors of $A_{0}$ and that they generate a $d$-dimensional subspace of $P^{*}$, invariant under $A$.

For each $y$ in $Q$, we have

$$
\psi_{\phi}^{i}(y)\left(x_{1}\right)=a_{i y} x_{1}
$$

for some element $a_{i y}$ in $K^{*}$. Thus $\phi^{-i} \psi_{y} \phi^{i}\left(x_{1}\right)=\psi_{\phi^{i}(y)}\left(x_{1}\right)=a_{i y} x_{1}$, so that $\psi_{y}\left(\phi^{i}\left(x_{1}\right)\right)=a_{i y} \phi^{i}\left(x_{1}\right)$, proving that $\phi^{i}\left(x_{1}\right)$ is a common characteristic vector of the elements of $\bar{Q}$. Since $\phi^{d j}$ and $\phi^{i}$ commute, $\phi^{i}\left(x_{1}\right)$ is also a characteristic vector of $\phi^{d j}$, and hence of every element of $A_{0}$.

Let $P^{*}{ }_{1}$ be the subspace of $P^{*}$ generated by the vectors $\phi^{i}\left(x_{1}\right)$. Since $\phi^{d}\left(x_{1}\right)=b_{1} x_{1}$ for some $b_{1}$ in $K^{*}, P^{*}$ is invariant under $A$; furthermore, the vectors $x_{1}, \phi\left(x_{1}\right), \ldots, \phi^{d}\left(x_{1}\right)$ are linearly dependent and hence $\operatorname{dim} P^{*}{ }_{1} \leqslant d$.

Suppose if possible that $\operatorname{dim} P^{*}{ }_{1}=k<d$. Then for $0 \leqslant i<k$ the vectors $\phi^{i}\left(x_{1}\right)$ are linearly independent, and furthermore

$$
\phi^{k}\left(x_{1}\right)=c_{0} x_{1}+c_{1} \phi\left(x_{1}\right)+\ldots+c_{k-1} \phi^{k-1}\left(x_{1}\right), c_{\jmath} \in K^{*},
$$

and $c_{0} \neq 0$. Apply $\psi_{y}$ to (4) and use (3) to obtain

$$
a_{k y} \phi^{k}\left(x_{1}\right)=c_{0} a_{0 y} x_{1}+c_{1} a_{1 y} \phi\left(x_{1}\right)+\ldots+c_{k-1} a_{k-1 y} \phi^{k-1}(y) .
$$

Now multiply (4) by $a_{k y}$ and subtract from (5), obtaining

$$
c_{0}\left(a_{0 y}-a_{k y}\right) x_{1}+c_{1}\left(a_{1 y}-a_{k y}\right) \phi\left(x_{1}\right)+\ldots+c_{k-1}\left(a_{k-1 y}-a_{k y}\right) \phi^{k-1}\left(x_{1}\right)=0 .
$$

Since $x_{1}, \phi\left(x_{1}\right), \ldots, \phi^{k-1}\left(x_{1}\right)$ are linearly independent and since $c_{0} \neq 0$, we conclude that

$$
a_{k y}=a_{0 y} .
$$

But (6) implies

$$
\phi^{-k} \psi_{y}^{-1} \phi^{k} \psi_{y}\left(x_{1}\right)=a_{0 y} \phi^{-k} \psi_{y}^{-1} \phi^{k}\left(x_{1}\right)=a_{0 y} a_{k y}^{-1} x_{1}=x_{1} .
$$

Thus $x_{1}$ is a common characteristic vector of all commutators $\phi^{-k} \psi_{y}^{-1} \phi^{k} \psi_{y}$, $y \in Q$, with the common characteristic root 1 . Since these linear transformations are defined over $K$ and 1 is in $K$, it is easy to show that they have a common characteristic vector $z_{1} \neq 0$ in $P$ with a common characteristic root 1 . But then

$$
\phi^{-k} \psi_{y}^{-1} \phi^{k} \psi_{y}\left(x_{1}\right)=\phi^{-k}\left(y\left(\phi^{k}\left(y^{-1} z_{1} y\right)\right) y^{-1}\right)=z_{1},
$$

and it follows that $\phi^{-k}(y) y^{-1}$ is in the centralizer of $\phi^{k}\left(z_{1}\right)$ for all $y$ in $Q$. But $Q$ is Abelian and hence the set of elements $\phi^{-k}(y) y^{-1}$ form a subgroup $Q_{0}$ of $Q$, which is clearly invariant under $\phi$. Since $k<d$ and $d$ is the order of $\phi$ 
on $Q, Q_{0} \neq 1$ and our hypotheses imply that $Q_{0}=Q$. Thus $\phi^{k}\left(z_{1}\right)$ commutes elementwise with $Q$, and since $P$ is Abelian, lies in the centre of $G$, contrary to the fact that $G$ has a trivial centre. Thus $\operatorname{dim} P^{*}{ }_{1}=d$, as asserted, and with respect to this basis, $\phi$ is represented on $P^{*}{ }_{1}$ by the companion matrix

$$
\Phi_{1}=\left(\begin{array}{lllllll}
0 & 1 & 0 & . & . & . & 0 \\
0 & 0 & 1 & . & . & . & 0 \\
& & & & & & \\
0 & 0 & 0 & . & . & . & 1 \\
b_{1} & 0 & 0 & . & . & . & 0
\end{array}\right) .
$$

Since $A_{0}$ is completely reducible and leaves $P^{*}$ invariant, we can write $P^{*}=P_{1} \oplus P^{\prime}$, where $P^{\prime}$ is invariant under $A_{0}$. If $P^{\prime} \neq 0$, we can construct as above a $d$-dimensional subspace $P^{*}{ }_{2} \subset P^{\prime}$, invariant under $A$, and with respect to a suitable basis of $P^{*}, \phi$ will be represented by a companion matrix $\Phi_{2}$, of the same form as $\Phi_{1}$, with possibly a different element $b_{2}$ in the $d$ th row, 1st column. Continuing this process, we can represent $P^{*}$ as the direct sum of subspaces $P^{*}{ }_{1}, P^{*}{ }_{2}, \ldots, P^{*}$, each invariant under $A$ and of dimension $d$, and with respect to a suitable basis of $P^{*}, \phi$ is represented by the matrix

$$
\phi=\left(\begin{array}{ccccc}
\Phi_{1} & & & & \\
& \Phi_{2} & & & \\
& & \cdot & & \\
& & & \cdot & \\
& & & \cdot & \\
& & & & \Phi_{\lambda}
\end{array}\right)
$$

where each $\Phi_{i}$ is a companion matrix of the form (7), having some element $b_{i}$ of $K^{*}$ in its $d$ th row, 1st column. In particular,

$$
m=d \lambda \text {. }
$$

From (8) we see that the characteristic polynomial of $\phi$ over $P^{*}$ is $g(x)=\left(x^{d}-b_{1}\right)\left(x^{d}-b_{2}\right) \ldots\left(x^{d}-b_{\lambda}\right)$ and that the characteristic polynomial of $\phi^{d}$ is $h(x)^{d}=\left[\left(x-b_{1}\right)\left(x-b_{2}\right) \ldots\left(x-b_{\lambda}\right)\right]^{d}$. Since $\phi$ is defined over $P$, the coefficients of $g(x)$ and hence of $h(x)$ are in $K$. A comparison with (2) now yields

$$
f(x)^{s}=h(x)^{d} .
$$

But $f(x)$ is irreducible, and hence $d \mid s$ and $h(x)=f(x)^{s / d}$. It follows that the roots $b_{1}, \ldots, b_{\lambda}$ of $h(x)$ are roots of $f(x)$ and hence lie in the field with $p^{t}$ elements. Since $t s=m$ and $d \mid s$ the quantities $b_{i}$ lie in the field with $p^{m / d}$ elements, and hence have orders dividing $p^{m / d}-1$. But by (8) $\phi^{d}$ is a diagonal matrix with $b_{1}, b_{2}, \ldots, b_{\lambda}$ as diagonal entries, and it follows that the order of $\phi^{d}$ divides $p^{m / d}-1$, which completes the proof of the lemma.

Lemma 3.2. If $G$ satsifies the hypotheses of the preceding lemma, let $F$ denote 
the set of elements of $G$ left fixed by $\phi^{r}$, for some fixed integer $r$. Then either $F \subset P$, $F=Q$, or $F=G$.

Proof. If $F \not \subset P$, there exists an element $z$ in $F$ with $z=x y, x$ in $P$, and $y \neq 1$ in $Q$. We have $x y=z=\phi^{r}(z)=\phi^{r}(x) \phi^{r}(y)$, whence $x \phi^{r}\left(x^{-1}\right)=y \phi^{r}\left(y^{-1}\right)$. Since the left side of this equation is an element of $P$, while the right is an element of $Q$, each is the identity, and so $\phi^{r}(y)=y$. Thus $y \in Q \cap F$, which is invariant under $\phi$. But $Q \cap F \neq 1$ and it follows from the hypotheses of Lemma 3.1 that $Q \cap F=Q$. Thus either $F \subset \mathrm{P}$ or $Q \subset F$.

Suppose now that $F>Q$, whence $F \cap P \neq 1$. If $x \in F \cap P, \phi^{r}\left(y x y^{-1}\right)$ $=\phi^{r}(y) \phi^{r}(x) \phi^{r}\left(y^{-1}\right)=y x y^{-1}$, and hence $y x y^{-1}$ is in $F \cap P$ for any $y$ in $Q$. Thus $F \cap P$ is normal in $G$, and being invariant under $\phi$, must equal $P$. Thus $F$ contains $P$ as well as $Q$, and we conclude that $F=G$.

4. $\phi$-groups of order $p^{m} q^{n}$. We shall need a preliminary lemma.

LEMMA 4.1. Let $G$ be an Abelian $\phi$-group of index $r$, of order $p^{m}$ and of type $(p, p, \ldots, p)$ and let $h$ be the order of $\phi$. Suppose $d|r, d| m$, and $h \mid d\left(p^{m / d}-1\right)$. Then either $d=1$ or $d=2, r \neq 0$, and the subgroup $F$ left elementwise fixed by $\phi^{r}$ has order $p$.

Proof. Let $s=m / d$. Since $o\left(\phi^{d}\right) \mid p^{s}-1, \phi^{d}$ is completely reducible when considered as a linear transformation, and each of its irreducible constituents has dimension $\leqslant s$. Thus $G$ is the direct product of subgroups $G_{1}, G_{2}, \ldots, G_{k}$ invariant under $\phi^{d}$, each of order $\leqslant p^{s}$ and $k \geqslant d$.

Let $g$ be a generator of $G$ under $\phi$ of index $r$ and write $g=g_{1} g_{2} \ldots g_{k}$, $g_{i} \in G_{i}, i=1,2, \ldots, k$. Since $G$ is Abelian, we have

$$
[g]_{r}^{j}=\prod_{i=1}^{d}\left[g_{i}\right]_{r}^{j} .
$$

Since $\phi^{d}$ leaves $G_{i}$ invariant and since $d \mid r$, it follows that $\left[g_{i}\right]_{\tau}^{j} \in G_{i}$ for all $i, j$.

Suppose first that $r=0$. Then $[g]_{r}^{p}=1$ and we have $h p>o(G)$, whence $d\left(p^{s}-1\right)>p^{s d-1}$, which implies $d=1$ or $d=2, s=1$, and $h=2(p-1)$.

On the other hand, if $r \neq 0$, the element

$$
\left[g_{i}\right]_{r}^{p^{8}-1}
$$

has order 1 or $p$ and is invariant under $\phi^{\tau}$, whence by (11) the same is true of

$$
[g]_{r}^{p^{s}-1} \text {. }
$$

It follows in either case that

$$
[g]_{r}^{p\left(p^{s}-1\right)}=1
$$

and hence that $h\left(p\left(p^{s}-1\right)\right)>o(G)$. Thus

$$
d\left(p^{s}-1\right)^{2}>p^{s d-1} .
$$

The only solutions of (12) are $d=1, d=2$, or $d=3, s=1$, and $h=3(p-1)$. 
In the third case the $G_{i}$ are cyclic of order $p$, for $i=1,2,3$ and are permuted cyclically by $\phi$. But then if the subgroup $F$ left elementwise fixed by $\phi^{r}$ were to contain some $G_{i}$, it would follow that $F=G$, whence $G$ would be of index 0 which is not the case. It follows that $F=1$ and hence that $[g]_{r}^{p-1}=1$. This leads, as in $(12)$, to the inequality $3(p-1)^{2}>p^{3}$, which is impossible.

We show next that $d=2, h=2\left(p^{s}-1\right)$ is impossible. In this case $G=G_{1} \otimes G_{2}$ where $G_{1}, G_{2}$ are invariant under $\phi^{2}$, of order $p^{s}$, and are permuted by $\phi$. If either $g_{1}=1$ or $g_{2}=1 \phi^{i}\left([g]_{r}{ }^{j}\right) \in G_{1} \cup G_{2}$, which is a proper subset of $G$. Thus we must have $g=g_{1} g_{2}$ with $g_{1} \neq 1, g_{2} \neq 1$. But now $\phi^{2}$ has order $p^{s}-1$ on both $G_{1}$ and $G_{2}$ and so $\left[g_{2}\right]_{r}^{j}=1$ implies $\left[g_{1}\right]_{\tau}{ }^{j}=1$. Thus the identity is the only element of $G_{1}$ which is of the form $\phi^{i}\left([g]_{r}{ }^{j}\right)$, contrary to the fact that $G$ is a $\phi$-group.

Suppose next that $d=2$ and $h<2\left(p^{s}-1\right)$. Since $h \mid 2\left(p^{s}-1\right)$, we conclude that $h<p^{s}-1$. But now $[g]_{r}^{h / r}=1$ implies $h^{2} / r>p^{2 s}$ which is clearly impossible. Thus $[g]_{r}{ }^{h / r}=x \neq 1$. Since $\phi^{r}(x)=x$, the subgroup $F$ left elementwise fixed by $\phi^{r}$ is not the identity. On the other hand, $[g]_{r}^{p h / r}=1$ and so $h(h / r) p>p^{2 s}$. It follows that $r<p$. Now $F$ is of index 0 , and hence every element of $F$ is of the form $\phi^{i}\left(y^{j}\right)$ for some element $y$ in $F$. But $\phi$ has order $r$ on $F$, and consequently $r p>o(F)$. Since $r<p$, we conclude that $F$ is cyclic, and the lemma is proved.

We are now ready to prove our main result concerning $\phi$-groups of order $p^{m} q^{n}$.

Lemma 4.2. If a $\phi$-group satisfies the conditions of Lemma 3.1, then $\phi$ leaves some element other than the identity fixed.

Proof. Let $g$ be a generator of $G$ under $\phi$ of index $r$, and let $F$ be the subgroup of $G$ of fixed elements under $\phi^{r}$. According to Lemma 3.2 either $F \subset P$, $F=Q$, or $F=G$.

Case 1 . F $\subset P$. Write $g=x y$, with $x$ in $P, y$ in $Q . P$ is normal in $G$, and hence $[g]_{r}^{j}=x_{j}[y]_{r}^{j}$ for some $x_{j}$ in $P$. If $t$ is the least integer such that $[y]_{r}{ }^{t}=1$, then $t$ is the least integer such that $[g]_{r}{ }^{t}$ is in $P$, and hence $P$ is a $\phi$-group of index $r t$. Moreover, since $Q$ is Abelian, it follows that $\phi^{r t}(y)=y$. But now the subgroup of $Q$ left fixed elementwise by $\phi^{r t}$ is invariant under $\phi$ and contains $y$, whence by our hypotheses it must equal $Q$. Thus the order $d$ of $\phi$ on $Q$ divides $r$, the index of $P$. In view of Lemma 3.1, $P$ now satisfies all the conditions of Lemma 4.1, and hence either $d=1$, in which case $\phi$ is the identity on $Q$, or $d=2$ and the subgroup $F_{1}$ of $P$ left elementwise fixed by $\phi^{r t}$ is cyclic.

In the latter case, $\phi^{r}$ leaves only the identity element of $Q$ fixed, since $F \subset P$, and hence $\phi^{r}$ has order 2 on $Q$. It follows that $\phi^{r}(z)=z^{-1}$ for all $z$ in $Q$. In particular this implies $t=2$. Furthermore if $\psi_{z}$ denotes the automorphism of $P$ induced by conjugation by an element $z$ in $Q$, we also have 
$\phi^{2 r} \psi_{z} \phi^{-2 r}=\psi_{z}$. If $F_{1}=\left(x_{1}\right)$, we conclude at once that $\phi^{2 r}\left(\psi_{z}\left(x_{1}\right)\right)=\psi_{z}\left(x_{1}\right)$, whence $\psi_{z}\left(x_{1}\right) \in F_{1}$ for all $z$ in $F_{1}$. Thus $F_{1}$ is normal in $G$, and being invariant under $\phi, F_{1}=P$, whence $o(P)=p$. Hence $m=1$, contrary to the fact that $d \mid m$ by Lemma 3.1 .

Case 2. $F=Q$. Since $F \neq G, r \neq 0$. If $r=1$, every element of $Q$ is left fixed by $\phi$. Hence we may assume $r>1$. We have $[y]_{r}^{q}=y^{q}=1$, and hence $x_{q}=[g]_{r}^{q} \in P$. Since $\phi^{\tau}$ is without non-trivial fixed elements on $P,\left[x_{q}\right]_{r}{ }^{h / r}=1$, $[g]_{r}{ }^{q h / r}=1$, and $h^{2} q>\operatorname{ro}(G)$ by Lemma 2.3. Since $h \mid d\left(p^{m / d}-1\right)$, we have

$$
d^{2}\left(p^{m / d}-1\right)^{2}>r p^{m} q^{n-1} .
$$

The only solutions of (13) are $d=1$, in which case the lemma follows, or $d=2$ and $r=2,3$. If $d=2, \phi^{2}$ leaves $Q$ elementwise fixed, while if $r=3$, $\phi^{3}$ leaves $Q$ elementwise fixed. Hence the case $d=2, r=3$ implies $\phi$ is the identity on $Q$. In the remaining case $d=2, r=2$, we have $d \mid r$ and hence by Lemma 4.1, the subgroup $F_{1}$ of $P$ left elementwise fixed by $\phi^{2 q}$ is cyclic (since $P$ is of index $2 q$ ). This leads to a contradiction as in Case 1 .

Case 3. $F=G$. This is the case $r=0 . P$ is also of index 0 , so that $d$ divides the index of $P$, whence by Lemma $4.1, d=1$. Thus $\phi$ is the identity on $Q$, and the lemma is established.

We wish to point out that there do exist $\phi$-groups satisfying the conditions of Lemma 3.1 in which $\phi$ leaves some non-trivial element of $G$ fixed. Perhaps the simplest example is the symmetric group $S_{3}$ on three letters, which can be defined by the relations $x^{3}=y^{2}=1$ and $y x y^{-1}=x^{-1}$. It is easily checked that $S_{3}$ is a $\phi$-group of index 1 with respect to the automorphism $\phi$ defined by: $\phi\left(x^{i} y^{j}\right)=x^{-i} y^{j}$, the element $x y$ being a generator of $S_{3}$ under $\phi$.

5. Solvable and non-exceptional $\phi$-groups. A group $G$ is called exceptional if $G$ is a non-cyclic simple group in which the normalizer of every characteristic subgroup $\neq 1$ of a $p$-Sylow subgroup $P$ of $G$ is $P$, for all primes $p \mid o(G)$. It is easily shown that if $G$ is solvable or if every Sylow subgroup of $G$ is Abelian, then no subgroup of $G$ has a composition factor which is an exceptional group (2, Lemma 4.1).

THEOREM 1. Let $G$ be a regular $\phi$-group and assume that no subgroup of $G$ has a composition factor which is an exceptional group. Then $G$ is nilpotent.

Proof. The proof is by induction on the order of $G$, and consists in reducing to the case in which $G$ satisfies the conditions of Lemma 3.1. This reduction is almost identical with that given by Feit (2, Lemma 4.2 and Theorem). However, as our group $G$ need not be the regular subgroup of a Frobenius group, we shall outline the steps in this portion of the proof.

We first show that $G$ contains a normal subgroup of prime power order invariant under $\phi$. If $G$ has a proper characteristic subgroup $H, H$ is nilpotent by induction, and any of its Sylow subgroups are normal in $G$ and invariant 
under $\phi$. Otherwise $G$ is the direct product of isomorphic non-exceptional simple groups. There exists then some $p \mid o(G)$ such that a $p$-Sylow subgroup $P$ of $G$ contains a characteristic subgroup $T$ such that $N(T)>P$. Since $\phi$ is a Frobenius automorphism, some $p$-Sylow subgroup of $G$ is invariant under $\phi$, and we may assume it to be $P$. Either $T$ is normal in $G$ (and $\phi(T)=T$ ) or by induction $N(T)$ is nilpotent, $P$ is normal in $N(T)$, and hence $N(P)>P$. Either the centre $C$ of $P$ is normal in $G$, and invariant under $\phi$ or $N(C)$ is nilpotent.

If neither $C$ nor $T$ is normal in $G$, we have $N(C) \supset N(P)>P$. If $Q$ is the unique $q$-Sylow subgroup of $N(C)$ for some prime $q \neq p$, and if $Q$ is not normal in $G, N(Q)$ is nilpotent and contains $P$, whence $P$ and $Q$ commute elementwise. If $C \supset x P x^{-1}$, then $Q \supset N\left(x^{-1} C x\right)$, which is nilpotent, so that $Q$ commutes elementwise with $x^{-1} P x$ as well as $P$. Since $N(Q)$ is nilpotent, $x^{-1} P x=P$, and it follows that $G$ is $p$-normal. But $N(P)$ is also nilpotent, so that by a theorem of Grün (6, p. 171) $G$ contains a normal subgroup $H$ such that $G / H \cong C$, contradicting the fact that $G$ is its own commutator subgroup. Thus $G$ contains a normal subgroup of prime power order, invariant under $\phi$.

Let $P$ be a minimal such subgroup so that $P$ is Abelian of type $(p, p, \ldots$, $p)$. By induction $G / P$ is nilpotent. If $G$ is not a $p$-group, suppose $q$ is a prime dividing $o(G), q \neq p$; and let $Q$ be a minimal subgroup invariant under $\phi$ of a $q$-Sylow subgroup of $G$. If $P Q<G, P Q$ is nilpotent and this, together with the fact that $G / P$ is nilpotent, implies that $Q$ is in the centre of $G$. But then $G / Q$ and hence $G$ is nilpotent.

We may suppose therefore that $G=P Q$, the centre of $G$ is trivial, no subgroup of $P$ invariant under $\phi$ is normal in $G$, and no subgroup of $Q$ is invariant under $\phi$-precisely the hypotheses of Lemma 3.1. But now Lemma 4.2 implies that there is no regular $\phi$-group which satisfies these conditions, and hence $G$ is nilpotent.

Corollary. If the Sylow subgroups of a regular $\phi$-group are $G$ Abelian, then $G$ is Abelian.

6. The fixed subgroup of $\phi^{r}$. The subgroup left elementwise fixed by $\phi^{r}$ plays an important role in determining the structure of a $\phi$-group of index $r$. In this section we shall determine some of the properties of this subgroup for $\phi$-groups of prime power order. We shall need the following lemma:

Leмма 6.1. Let $G$ be a $\phi$-group of index 0 of order $p^{a}$ having a generator $g$ of order $p^{n}$. Then $G$ contains a sequence of characteristic subgroups $G=G_{n} \supset$ $G_{n-1} \supset \ldots \supset G_{1} \supset G_{0}=1$ where $G_{i}$ is generated by the elements of order $p^{i}$ in $G$. Moreover, the subgroups $G_{i}$ are the only subgroups of $G$ invariant under $\phi$.

Proof. Since $G$ is a $\phi$-group of index 0 , the elements $\phi^{i}\left(g^{p^{n-1} j}\right)$ clearly include all elements of order $p$ in $G$. Since no proper subset of these elements form a 
subgroup invariant under $\phi$, they must form the characteristic subgroup $G_{1}$ of elements of order dividing $p$ in the centre of $G$. As pointed out, no proper subgroup of $G_{1}$ is invariant under $\phi$.

The lemma follows now easily by applying induction to the group $G / G_{1}$.

Theorem 2. Let $G$ be a regular $\phi$-group of index $r$ and order $p^{a}$, and let $F$ be the subgroup of $G$ left elementwise fixed by $\phi^{r}$. Then every subgroup of $F$ invariant under $\phi$ is normal in $G$.

Proof. Since $\phi^{r}$ leaves $F$ elementwise fixed, $F$ is of index 0 , and hence by the preceding lemma the elements of order $p$ in $F$ form a characteristic subgroup $F_{1}$ of $F$. If $F_{1}$ is normal in $G$, the theorem follows by induction. For if we set $\bar{G}=G / F_{1}, \bar{F}=$ the residue of $F$ in $\bar{G}$, and $\bar{F}^{\prime}$ the subgroup of elements left elementwise fixed by the image $\bar{\phi}^{r}$ of $\phi^{r}, \bar{F} \subset \bar{F}^{\prime}$ and $\bar{F}^{\prime}$ is normal in $\bar{G}$ by induction. Since $\bar{F}$ is invariant under $\bar{\phi}, \bar{F}$ is characteristic in $\bar{F}^{\prime}$ by the preceding lemma, and hence normal in $\bar{G}$. Thus $F$ is normal in $G$ and the theorem follows at once.

We shall actually prove that $F_{1}$ lies in the centre of $G$. Let $h$ be the order of $\phi$, let $g$ be a generator of $G$ under $\phi$, and let

$$
g_{1}=[g]_{\tau}^{h_{1} / r}
$$

be a generator of $F_{1}$, so that $F_{1}$ is of index $h_{1}$. To our induction hypothesis we shall add the assertion that either $h / h_{1}$ or $h_{1} / h$ is a power of $p$.

Let us begin by verifying this statement under the assumption that $F_{1}$ is in the centre of $G$. Let $k$ be the order of $\bar{\phi}$ of $\bar{G}$ and let $\bar{g}$ be the residue of $g$ in $\bar{G}$. Let $H$ be the set of elements of $G$ left fixed by $\phi^{k}$ and suppose first the $H \not \supset F_{1}$. Then $H \cap F_{1}=1$ and hence $\phi^{k}$ is Frobenius on $F_{1}$. Thus $\phi^{k}(g)=x g$, $x \in F_{1}$ and $x=y^{-1} \phi^{k}(y)$ for some $y$ in $F_{1}$. It follows that $\phi^{k}\left(g y^{-1}\right)=g y^{-1}$, whence $g y^{-1} \in H$. Thus $g \in F_{1} H$. Since $F_{1} H$ is invariant under $\phi$ and contains $g, G=F_{1} H$. Since $H \cong \bar{G}, \phi$ has order $k$ on $H$. If $(r, k)=s$, it follows that

$$
h=\frac{r k}{s} .
$$

On the other hand, let $H_{1}$ be the subgroup of $H$ generated by the elements of order $p$ left elementwise fixed by $\phi^{r}$. Then $F_{1} H_{1}$ is left elementwise fixed by $\phi^{r}$ and its elements all have order dividing $p$. It follows that $F_{1} H_{1}=F_{1}$. Since $F_{1} \cap H_{1}=1$, we conclude that $H_{1}=1$. Hence $\phi^{r}$ leaves only the identity element of $H$ fixed, and consequently $\bar{\phi}^{r}$ leaves only the identity element of $\bar{G}$ fixed. But this implies $k / s$ is the least integer such that $[\bar{g}]_{r}{ }^{k / s}=1$, and hence $g_{1}=[g]_{r}^{k / s}$. Thus $r k / s$ is the index of $F_{1}$, and in view of (14) we conclude that $h_{1}=h$.

Hence we may suppose $H \supset F_{1}$. In this case, the relation $\phi^{k}(g)=x g$ implies $\phi^{k p}(g)=g$, and we have

$$
k|h| k p \text {. }
$$


Since $r \mid h$, it follows that either $r=s$ or $r=s p$. If $\bar{\phi}^{r}$ leaves only the identity element of $\bar{G}$ fixed, it follows as above that $h_{1}=k r / s$, and hence $k\left|h_{1}\right| k p$. We conclude from (15) that either $h / h_{1}$ or $h_{1} / h$ is a power of $p$.

If $\bar{\phi}^{r}$ is not Frobenius, let $\bar{F}_{1}$ be the subgroup of $\bar{G}$ generated by the elements of order $p$ left elementwise fixed by $\bar{\phi}^{r}$. If $k_{1}$ is the index of $\bar{F}_{1}$, then by induction either $k / k_{1}$ or $k_{1} / k$ is a power of $p$. By definition of $k_{1}$,

$$
[\bar{g}]_{\tau}^{k_{1} / \tau}
$$

is a generator of $\bar{F}_{1}$, and hence

$$
g_{2}=[g]_{\tau}^{k_{1} / r}
$$

is a generator of the inverse image $F_{2}$ of $\bar{F}_{1}$. Since $\bar{\phi}^{r}$ leave $\bar{F}_{1}$ elementwise fixed and $r \mid k_{1}$,

$$
\phi^{k_{1}}\left(g_{2}\right)=z g_{2}
$$

for some $z$ in $F_{1}$. Since $F_{1}$ is in the centre of $F_{2}$, this implies

$$
\left[g_{2}\right]_{k_{1}}^{j}=z^{j(j-1) / 2} g_{2}^{j} .
$$

As $p$ is the least power of $j$ for which $g_{2}{ }^{p} \in F_{1}$, it follows at once that $h_{1}=k_{1} p$. Thus $h_{1}=k p^{\epsilon}$ for some integer $\epsilon$. This together with (15) implies that either $h / h_{1}$ or $h_{1} / h$ is a power of $p$.

Finally we must show that $F_{1}$ does in fact lie in the centre of $G$. Let $C$ be a minimal subgroup of the centre of $G$ invariant under $\phi$. Because of the minimality of $F_{1}$, either $C=F_{1}$ or $C \cap F_{1}=1$. In the latter case, let $\widetilde{G}, \widetilde{F}_{1}$, $\widetilde{g}, \tilde{\phi}$ be respectively $G / C$, the image of $F_{1}$ and $g$ in $G / C$, and the image of $\phi$ on $G / C$. Let $m$ be the order of $\widetilde{G}$ on $\widetilde{F}$, and define $M$ to be the subgroup of $G$ left elementwise fixed by $\phi^{m}$.

Now by induction, if $m_{1}$ is the index of $\bar{F}_{1}$, we have

$$
\frac{m}{m_{1}}=p^{\epsilon}
$$

for some integer $\epsilon$. that

By definition of $m_{1}, r \mid m_{1}$. If we write $r=r_{1} p^{\delta}$, where $\left(r_{1}, p\right)=1$, it follows

$$
r_{1} \mid m \text {. }
$$

Since every element of $F_{1}$ is of the form $\phi^{i}\left(g_{1}{ }^{j}\right)$, the order of $\phi$ on $F_{1}$ is relatively prime to $p$, and hence $\phi^{r_{1}}$ leaves $F_{1}$ elementwise fixed. It follows therefore from (18) that $F_{1} \subset M$.

Assume first that $C \subset M$, in which case $C F_{1} \subset M$. Now the index of $C F_{1}=$ index of $\bar{F}_{1}=m_{1}$. Let $g^{\prime}$ be a generator of $C F_{1}$ of index $m_{1}$ and write $g^{\prime}=x y$, $x \in C, y \in F_{1}$. If $m \mid m_{1}$,

$$
\left[g^{\prime}\right]_{m_{1}}^{j}=g^{\prime j}=x^{j} y^{j}
$$


and every element of $C F_{1}$ is of the form $\phi^{i}\left(x^{j} y^{j}\right)$, which is clearly impossible since $C \cap F_{1}=1$. On the other hand, if $m \nmid m_{1}$ (17) holds with $\epsilon \supset 0$, and in this case

$$
\left[g^{\prime}\right]_{m_{1}}^{j}=[x]_{m_{1}}^{j} y^{j} .
$$

To obtain an element of $F_{1}$, we must have

$$
[x]_{m_{1}}^{j}=1 \text {, }
$$

and this implies $\phi^{m_{1} j}(x)=x$ since $C$ is Abelian. If $j=1$,

$$
\left[g^{\prime}\right]_{m_{1}}^{j}=x^{j} y^{j}
$$

which is impossible as above. Since

$$
\phi^{m_{1} p^{\epsilon}}(x)=x,
$$

$j \neq 1$ implies $p \mid j$ and hence 1 is the only element of $F_{1}$ which can be written in the form

$$
\phi^{i}\left(\left[g^{\prime}\right]_{m_{1}}^{j}\right),
$$

contrary to the fact that $g^{\prime}$ is a generator of $C F_{1}$ under $\phi$.

On the other hand, if $C \cap M=1$, it follows as in an earlier part of the proof that $G=C M$. But $M \prec G$ and $F_{1} \subset M$ so that by induction $F_{1}$ is in the centre of $M$. Since $C$ is in the centre of $G$, it follows that $F_{1}$ is in the centre of $G$, and the proof is complete.

Corollary. If $F_{1}$ denotes the subgroup of $F$ generated by the elements of order $p$ in $F$, then $F_{1}$ lies in the centre of $G$.

7. $\phi$-groups in which $\phi^{r}$ leaves only the identity fixed. We shall also need some properties of $\phi$-groups of index $r$ in which $\phi^{r}$ is a Frobenius automorphism. To this end, we first establish the following lemma.

Lemma 7.1. Let $G$ be a regular $\phi$-group of prime power order, and let $C$ be a subgroup of the centre of $G$, invariant under $\phi$ and of least possible order. Then either $C=G$ or $[o(C)]^{2} \leqslant o(G)$.

Proof. We may suppose $G>C$. If $\bar{G}=G / C$, we may restrict our attention to a minimal subgroup of the centre of $\bar{G}$, and hence without loss of generality we may assume that $\bar{G}$ is Abelian of type $(p, p, \ldots, p)$ and that no proper subgroup of $\bar{G}$ is invariant under the image $\bar{\phi}$ of $\phi$ on $\bar{G}$.

Let $g$ be a generator of $G, \vec{g}$ its image in $\bar{G}, k$ the order of $\bar{\phi}$, and $H$ the subgroup of $G$ left elementwise fixed by $\phi^{k}$. If $H \cap C=1$, it follows as in the preceding section that $G=C H$. Since $G / C \cong H, H$, and hence $G$, is Abelian. But by definition of $C, o(C) \leqslant o(H)$ and therefore $[o(C)]^{2} \leqslant o(G)$.

If, on the other hand, $H \supset C$, the equation $\phi^{k}(g)=y g$ implies $\phi^{k p}(g)=g$ so that $h \mid k p$, where $h$ is the order of $\phi$. If $h=k$ and $\phi^{r}$ leaves only the identity element fixed, it follows as in the preceding section that the identity is the 
only element $C$ which can be written in the form $\phi^{i}\left([g]_{r}^{\jmath}\right)$, which is a contradiction.

If $h=k$ and some proper subgroup $F$ of $G$ is left elementwise fixed by $\phi^{k}$, either $F \cap C=1$ or $F \supset C$. In the first case, since no proper subgroup of $\bar{G}$ is invariant under $\bar{\phi}$, it follows that $G=C F$, and hence $G$ is Abelian since $F \cong \bar{G}$ is Abelian; and we have $[o(C)]^{2} \leqslant o(G)$.

If $F>C$, then $F=G$ is of index 0 , and hence $C$ contains all elements of $G$ of order $p$. If $\bar{x}_{1}, \bar{x}_{2}, \ldots, \bar{x}_{m}$ are a basis of $\bar{G}$, let $x_{1}, x_{2}, \ldots, x_{m}$ be a set of representatives such that $\phi\left(x_{i}\right)=x_{i+1}, i=1,2, \ldots, m-1$. Then

$$
\phi\left(x_{m}\right)=z x_{1}^{\alpha_{1}} x_{2}^{\alpha_{2}} \ldots x_{m}^{\alpha_{m}} .
$$

Since $x_{i}{ }^{p} \in C$ for all $i$, it follows at once that $x_{1}{ }^{p}, x_{2}{ }^{p}, \ldots, x_{m}{ }^{p}$ generate a subgroup $C_{1}$ of $C$ invariant under $\phi$. Since $C$ is minimal, $C_{1}=C$, and hence $o(C) \leqslant p^{m}=o(\bar{G})$, which implies $[o(C)]^{2} \leqslant o(G)$.

Finally if $F=C, C$ is of index 0 , whence the order of $\phi$ on $G$ is a multiple of $p^{n}-1 / p-1$, where $p^{n}=o(C)$. This implies $\left(p^{n}-1\right) /(p-1) \mid k$. But $\bar{G}$ is an Abelian group of type $(p, p, \ldots, p)$ and hence $k<o(\bar{G})$. Thus $o(C)$ $=p^{n} \leqslant o(\bar{G})$ and $[o(C)]^{2} \leqslant o(G)$, as desired.

We now prove

THEOREM 3. Let $G$ be a regular $\phi$-group of index $r$ and assume $\phi^{r}$ leaves only the identity element of $G$ fixed. Then either some Sylow subgroup of $G$ is Abelian or there exists a proper subgroup $G_{1}$ in $G$, invariant under $\phi$, which contains a non-trivial subgroup of the centre of some $p$-Sylow subgroup of $G$ for every prime $p \mid o(G)$.

Proof. If $g$ is a generator of $G$ under $\phi$, and if $h$ denotes as usual the order of $\phi$, we have first of all $[g]_{r}{ }^{h / r}=1$ and hence by Lemma 2.3

$$
h^{2}>o(G) \text {. }
$$

Let $p_{1}, \ldots, p_{t}$ be the distinct primes dividing $o(G)$, and let $P_{1}, \ldots, P_{t}$ be the corresponding Sylow subgroups of $G$ invariant under $\phi$. Let $C_{i}$ be a minimal subgroup of $P_{i}$, invariant under $\phi$, and of lowest possible order. Then if no Sylow subgroup of $G$ is Abelian, the preceding lemma gives

$$
\left[o\left(C_{i}\right)\right]^{2} \leqslant o\left(P_{i}\right), \quad i=1,2, \ldots, t .
$$

Define $s_{i}$ by the condition that

$$
g_{i}=[g]_{r}^{s i}
$$

be a generator of $C i$, and let $h_{i}$ be the order of $\phi$ on $C_{i}, i=1,2, \ldots, t$. Since $C_{i}$ is an Abelian group of type $\left(p_{i}, p_{i}, \ldots, p_{i}\right)$, we have

$$
h_{i}<o\left(C_{i}\right), \quad i=1,2, \ldots, t .
$$

Now let $\lambda$ be the greatest common divisor of $s_{1}, s_{2}, \ldots, s_{t}$. We may assume the $s_{i}$ are so numbered that 


$$
\lambda=\sum_{i=1}^{m} a_{i} s_{i}-\sum_{i=m+1}^{t} b_{i} s_{i}, \quad \text { where } a_{i}, b_{i} \geqslant 0 .
$$

We now consider the elements

$$
\begin{aligned}
& x=\left[g_{1}\right]_{r s_{1}}^{a_{1}} \phi^{r a_{1} s_{1}}\left(\left[g_{2}\right]_{r s_{2}}^{a_{2}}\right) \ldots \phi^{\tau\left(a_{1} s_{1}+\ldots+a_{m-1} s_{m-1}\right)}\left(\left[g_{m}\right]_{r s_{m}}^{a_{m}}\right) \\
& y=\left[g_{m+1}\right]_{r s_{m+1}}^{b_{m+1}} \phi^{r b_{m+1} s_{m+1}}\left(\left[g_{m+2}\right]_{r s_{m}+2}^{b_{m+2}}\right) \ldots \phi^{r\left(b_{m}+1 s_{m+1}+\ldots+b_{t-1} s_{t-1}\right.}\left(\left[g_{t}\right]_{r s_{t}}^{b_{t}}\right) .
\end{aligned}
$$

By repeated use of Lemma 2.1, we find that

$$
x=[g]_{r}^{u} \text { and } y=[g]_{r}^{v}, \quad \text { where } u=\sum_{i=1}^{m} a_{i} s_{i}, v=\sum_{i=m+1}^{t} b_{i} s_{i},
$$

and hence that $z=y^{-1} x=\phi^{r v}(g) \phi^{r(v+1)}(g) \ldots \phi^{r(u-1)}(g)$. It follows that

$$
z=\phi^{r v}\left([g]_{r}^{\lambda}\right) .
$$

By construction $z$ is a power product of elements of $C_{1}, C_{2}, \ldots, C_{t}$, and hence we have $\phi^{k}(z)=z$ for some integer $k \mid \Pi_{1} h_{i}$. Therefore

$$
\phi^{k}\left([g]_{r}^{\lambda}\right)=[g]_{r}^{\lambda} \quad \text { with } \quad k \mid \prod_{i=1}^{t} h_{i} .
$$

Now let $G_{1}$ be the subgroup of $G$ invariant under $\phi$ which is generated by $[g]_{r}^{\lambda}$. We prove that $G_{1}$ is a proper subgroup of $G$. Suppose, on the contrary, that $G_{1}=G$. Then $\phi^{k}$ is the identity on $G$ by (27) and hence $h \mid k$.

But then combining (21), (22), and (23), we get

$$
h^{2} \leqslant \prod_{i=1}^{t} h_{i}^{2}<\prod_{i=1}^{t}\left[o\left(C_{i}\right)\right]^{2} \leqslant \prod_{i=1}^{t}\left(o\left(P_{i}\right)\right)=o(G),
$$

in contradiction to $(20)$.

Since $\lambda \mid s_{i}$ for all $i,[g]_{r}{ }^{s i}$ and hence $C_{i}$ is contained in $G_{1}$ for all $i=1,2, \ldots$, $t$, and the theorem is proved.

COROLlary. The same conclusion holds if we assume that $h^{2} / r>o(G)$ instead of that $\phi^{r}$ leaves only the identity element of $G$ fixed.

8. The structure of regular $\phi$-groups. We are now in a position to prove our main result

THEOREM 4. Every regular $\phi$-group is nilpotent.

Proof. Let $G$ be a regular $\phi$-group of index $r, g$ a generator of $G$ under $\phi$, and let $k$ be the least integer such that $[g]_{r}{ }^{k}=1$. The proof will be by induction on $k$.

If $H$ is a proper subgroup of $G$ invariant under $\phi$, and $s$ the least integer such that $z=[g]_{r}^{s} \in H$, then clearly $s \mid k, z$ is a generator of $H$ of index $r s$ and $[z]_{r s}{ }^{k / s}=1$. Hence by induction $H$ is nilpotent.

It suffices therefore, in view of Theorem 1, to prove that the normalizer 
of a characteristic subgroup of some Sylow subgroup $P$ of $G$ contains $P$ properly. As in Theorem 1, we may suppose $G$ contains no proper characteristic subgroup; and hence that $G$ is the direct product of isomorphic non-cycle simple groups, no subset of which is invariant under $\phi$.

Let $p_{1}, p_{2}, \ldots, p_{t}$ be the distinct primes dividing $o(G)$, and let $P_{1}, P_{2}$, $\ldots, P_{t}$ be the corresponding Sylow subgroups of $G$ invariant under $\phi$. If, first of all, some $P_{i}$ is Abelian, $N\left(P_{i}\right) \prec G$, and hence is nilpotent. Thus $P_{i}$ is in the centre of its normalizer, and it follows by a theorem of Burnside that $G$ contains a normal subgroup $H$ such that $G / H \cong P_{i}$, contrary to the fact that $G$ is its own commutator subgroup.

Thus no Sylow subgroup of $G$ is Abelian. If $\phi^{r}$ left only the identity element of $G$ fixed, it would follow from Theorem 3 that there exists a proper subgroup $G_{1}$ in $G$, invariant under $\phi$ which contains for each $i=1,2, \ldots, t$ a subgroup $C_{i}{ }^{\prime}$ of the centre of $P_{i}$. Since $G_{1}$ is nilpotent by induction and $G$ is not a p-group, $N\left(C_{i}{ }^{\prime}\right)>P_{i}$. Now $N\left(C_{i}{ }^{\prime}\right)<G$ and so is nilpotent. If $C_{i}$ denotes the centre of $P_{i}$, it follows that $N\left(C_{i}\right)>P_{i}, i=1,2, \ldots, t$, and by a previous remark this is sufficient to prove the nilpotency of $G$. Hence if $F$ denotes the subgroup of $G$, left elementwise fixed by $\phi^{r}, F>1$.

Let $g_{i}=[g]_{r}^{s_{i}}$ be a generator of $P_{i}, i=1,2, \ldots, t$ and define $F_{i}$ to be the subgroup of $G$ left elementwise fixed by $\phi^{r s_{i}}$. Clearly $F \subset F_{i}$ for all $i$. Suppose first there is an index $i$, say $i=1$, for which $o\left(F_{1}\right)$ is divisible by at least two distinct primes.

If $F_{1} \prec G$, then $F_{1}$ is nilpotent by induction. Let $P_{i}^{\prime}$ be the $p_{i}$-Sylow subgroup of $F$ invariant under $\phi$. As is easily seen, $P_{i}{ }^{\prime} \subset P_{i}$. Suppose for some $i$ $p_{i} \mid o(F)$. By Theorem 2, $F_{i} \cap P_{i}$ is normal in $P_{i}$ and $F \cap P_{i}$ being invariant under $\phi$, is a characteristic subgroup of $F_{i} \cap P_{i}$, and hence is normal in $F_{i} \cap P_{i}$ Thus $V\left(F \cap P_{i}\right) \supset P_{i}$ Since $F_{1}$ is nilpotent, it follows that $F \cap P_{i}$ is normal in $F_{1}$, and hence $N\left(F \cap P_{i}\right) \supset F_{1}$. It follows from our assumption on $o\left(F_{1}\right)$ that $N\left(F \cap P_{i}\right)>P_{i}$. Since $N\left(F \cap P_{i}\right)$ is nilpotent by induction, we conclude that $N\left(C_{i}\right)>P_{i}$ which is sufficient to prove the nilpotency of $G$.

Suppose instead that $F_{1}=G$, so that $\phi^{r s_{1}}$ is the identity on $G$. If $g_{1}$ has order $p^{n}$, it follows that

$$
\left[g_{1}\right]_{r s_{1}}^{p^{n}}=g_{1}^{p^{n}}=1 \text {, whence }[g]_{r}^{s_{1} p^{n}}=1,
$$

and consequently

$$
k=s_{1} p^{n} .
$$

Since $G$ is not solvable, the well-known theorem of Burnside implies $t \geqslant 3$. It follows from (29) that $s_{2} \mid s_{1} p^{n}$ and $s_{3} \mid s_{1} p^{n}$. However $s_{2} \nmid s_{1}$, for this would imply that $[g]_{r}^{s_{1}} \in P_{1} \cap P_{2}=1$, which is not the case. Similarly $s_{3} \nmid s_{1}$, and hence

$$
p_{1}\left|s_{2}, \quad p_{1}\right| s_{3} .
$$

Let $G_{1}$ be the subgroup generated under $\phi$ by

$$
g^{\prime}=[g]_{\tau}^{p_{1}}
$$


By (30), $G_{1} \supset P_{2}$ and $G_{1} \supset P_{3}$. If $G_{1}<G, G_{1}$ is nilpotent by induction, and consequently $N\left(C_{2}\right)>P_{2}$, from which the nilpotency of $G$ follows. On the other hand, if $G_{1}=G, g^{\prime}$ is a generator of $G$ under $\phi$ of index $r p_{1}$ and

$$
\left[g^{\prime}\right]_{\tau p_{1}}^{k^{\prime}}=1 \text {, where } k^{\prime}=k / p_{1} \text {. }
$$

Since $k^{\prime}<k$, the nilpotency of $G$ follows by induction.

Finally we must consider the case in which each $F_{i}$ is of prime power. Since $F \subset F_{i}$ for all $i, o\left(F_{i}\right)$ is a power of a single prime, say $p_{1}$, for all $i=1,2, \ldots, t$. In particular, this implies $F_{i} \cap P_{i}=1, i=2,3, \ldots, t$, and $\phi^{r s_{i}}$ leaves only the identity element of $P_{i}$ fixed. Once again $t \geqslant 3$.

It follows at once from the fact that $[g]_{r}{ }^{k}=1$ that $\phi^{r k}(g)=g$, and hence that $(h / r) \mid k$. Thus

$$
k=m h / r
$$

for some integer $m$.

If $m=1, h(h / r)>o(G)$, and it follows from the corollary of Theorem 3 that either some Sylow subgroup of $G$ is Abelian or $G$ contains a proper subgroup $G_{1}$ satisfying the conditions of Theorem 3 . Since both of these cases have been treated above, we may assume $m>1$.

On the other hand, since $\phi^{r s_{i}}$ leaves only the identity element of $P_{i}$ fixed,

and hence

$$
\left[g_{i}\right]_{r i}^{h / r}=1, \quad i=2,3
$$

It follows that

$$
[g]_{r}^{h s_{i} / r}=1
$$

If now $G^{*}{ }_{1}$ is the subgroup of $G$ generated under $\phi$ by $g^{*}=[g]_{r}{ }^{m}, G^{*}{ }_{1} \supset P_{2}$ and $G^{*}{ }_{1} \supset P_{3}$ in view of $(32)$. If $G^{*}{ }_{1}<G$, it follows as above that $N\left(C_{2}\right)>P_{2}$ and that $G$ is nilpotent; while if $G^{*}{ }_{1}=G, g^{*}$ is a generator of $G$ of index $r m$,

$$
\left[g^{*}\right]_{r m}^{k^{*}}=1
$$

where $k^{*}=k / m$, and $G$ is nilpotent by induction.

9. The solvability of $\phi$-groups. We now prove

THEOREM 5. Every $\phi$-group is solvable.

Proof. Let $G$ be a $\phi$-group of index $r$ with respect to a generator $g$, and let $h$ be the order of $\phi$. As in $\S 2$, we imbed $G$ as a normal subgroup of a group $G^{*}$ which satisfies the following conditions:

$$
G^{*}=G A \text { with } G \cap A=1, a y a^{-1}=\phi(y)
$$

for some element $a$ in $G^{*}$ of order $h$ and all $y$ in $G$.

If

$$
y=\phi^{i}\left([g]_{\tau}^{j}\right)
$$


is an arbitrary element of $G$, we can represent $y$ in $G^{*}$ in the form

$$
y=a^{i}\left[g\left(a^{\tau} g a^{-\tau}\right)\left(a^{2 r} g a^{-2 r}\right) \ldots\left(a^{(j-1) r} g a^{-(j-1) r}\right) a^{-i},\right.
$$

which reduces to

$$
y=a^{i}\left(g a^{r}\right)^{j} a^{-j r-i}
$$

Setting $b=g a^{r}$, every element of $G$ can thus be expressed in the form $a^{i} b^{j} a^{-j r-i}$ for suitable choice of $i$ and $j$. If $x \in G^{*}, x=y a^{k}$ for some $y$ in $G$ and some integer $k$. It follows that

$$
\text { if } x \in G^{*}, x=a^{u} b^{v} a^{w} \text { for suitable integers } u, v, w \text {. }
$$

If $\phi$ leaves only the identity element of $G$ fixed, $G$ is nilpotent by Theorem 4; and so we may assume that there is a subgroup $H \neq 1$ in $G$ which is left elementwise fixed by $\phi$.

Let $g_{1}=[g]_{r} s$ te a generator of $H$, so that by (34)

$$
g_{1}=b^{s} a^{-r s} \text {. }
$$

Since $\phi\left(g_{1}\right)=g_{1}$, we have $a g_{1} a^{-1}=a b^{s} a^{-r s} a^{-1}=b^{s} a^{-r s}$, whence

$$
a b^{s} a^{-1}=b^{s} \text {. }
$$

Thus $b^{s}$ commutes with $a$. Since $b^{s}$ obviously commutes with $b$, it follows from (35) that $b^{s}$ is in the centre of $G^{*}$. Let $C^{*}$ be the subgroup generated by $b^{s}$, and set $\bar{G}^{*}=G^{*} / C^{*}$. Denoting the images of $a, b, g, G$ in $\bar{G}^{*}$ respectively by $\bar{a}, \bar{b}, \bar{g}, \bar{G}$, it follows first of all that $\bar{G}$ is normal in $G^{*}$, and secondly that every element of $\bar{G}$ is of the form $\bar{a}^{i} \bar{b}^{j} \bar{a}^{-j r-i}$, while every element of $\bar{G}^{*}$ is of the form $\bar{a}^{u} \bar{b}^{v} \bar{a}^{w}$. If $\bar{\phi}$ denotes the automorphism of $\bar{G}$ induced by conjugation by $\bar{a}$, we can reverse the steps in the derivation of (34) to conclude that every element of $\bar{G}$ is of the form $\bar{\phi}^{i}\left([\bar{g}]_{r}^{j}\right)$. Thus $\bar{G}$ is a $\bar{\phi}$-group; and by definition of $\bar{\phi}$, we have

$$
\bar{\phi}(\bar{y})=\bar{a} \bar{y} \bar{a}^{-1}, \quad \bar{y} \in \bar{G} .
$$

Either $\bar{\phi}$ leaves only the identity element of $\bar{G}$ fixed or we may repeat the process. Continuing this process we can always construct a sequence of groups

$$
G_{i}^{*}, i=1,2, \ldots, n \quad \text { with } \quad G^{*}=G_{1}^{*}, \quad \tilde{G}^{*}=G_{2}^{*},
$$

satisfying the following conditions:

(1) $G_{i+1}^{*}=G_{i}^{*} / C_{i}^{*}$, where $C_{i}^{*}$ is a cyclic subgroup of the centre of $G_{i}^{*}$, $i=1,2, \ldots, n-1$;

(2) $G_{n}^{*}$ is either the identity or contains a normal subgroup $G_{n}$ such that $G / G_{n}$ is cyclic;

(3) $G_{n}$ is a $\phi_{n}$-group in which $\phi_{n}$ leaves only the identity element of $G_{n}$ fixed. 
By Theorem $4, G_{n}$ is nilpotent. Hence $G^{*}{ }_{n}$ and conseqently $G^{*}$ is solvable. Since $G \subset G^{*}$, it follows that $G$ is solvable.

Remark. Not every $\phi$-group is nilpotent. An example of a non-nilpotent $\phi$-group is the symmetric group on 3 letters, which was discussed at the end of $\S 4$.

10. $\phi$-groups of index 0 . In the next two sections we shall show that a regular $\phi$-group of prime power order is either Abelian or metabelian. In view of Theorem 4 this will imply that a regular $\phi$-group is nilpotent of class $\leqslant 2$.

In (4, Lemma 2), it has been shown that a regular $\phi$-group of index 0 is Abelian if the order of $\phi$ is relatively prime to the order of a generator of $G$ under $\phi$. In this section we shall establish the same result without making any restrictions on the order of $\phi$.

We have seen in Lemma 6.1 that a $\phi$-group $G$ of index 0 and of prime power order contains a sequence of subgroups $G=G_{n} \supset G_{n-1} \supset \ldots \supset G_{1} \supset G_{0}=1$, where the $G_{i}$ are the only subgroups of $G$ invariant under $\phi$, where each $G_{i}$ is normal in $G$, and where $x^{-1} \phi^{r}(x)=1$ if $x \in G_{i}, i=0,1,2, \ldots, n$. For later purposes we need to investigate $\phi$-groups of prime power order which contain such a sequence of subgroups $G_{i}$ satisfying the first two of these conditions together with following weaker third conditions: if $x \in G_{i}$, then

$$
x^{-1} \phi^{r}(x) \in G_{i-1}, \quad i=0,1,2, \ldots, n .
$$

We begin with the following lemma.

Lemma 10.1. Let $G$ be an Abelian $\phi$-group of index $r$, of order $p^{n m}$ and type $\left(p^{n}, p^{n}, \ldots, p^{n}\right)$. Denote by $G_{i}$ the subgroup generated by the elements of order $p^{i}$, and assume that for every $x$ in $G_{i}, x^{-1} \phi^{\tau}(x)$ is in $G_{i-1}$. Then if $h$ is the order of $\phi$, we have $h \mid p^{n-1}\left(p^{m}-1\right)$ and either $n=1$ or $m \leqslant 2$.

Proof. Let $g$ be a generator of $G$ under $\phi$. If $n=1, G$ is of order $p^{m}$, of type $(p, p, \ldots, p)$, and $g^{-1} \phi^{\tau}(g)=1$, so that $G$ is of index 0 , and every element of $G$ is of the form $\phi^{i}\left(g^{j}\right)$. Hence the orbit under $\phi$ of $g^{j}$ contains exactly $h$ elements, if $0<j<p$; if the number of distinct such orbits is $k$, we have $h k+1=p^{m}$, whence $h \mid p^{m}-1$.

If $n>1$, we proceed by induction to prove the first part of the lemma. $G_{n-1}$ is of type $\left(p^{n-1}, p^{n-1}, \ldots, p^{n-1}\right)$, of order $p^{(n-1) m}$, and is invariant under $\phi$. Since $\phi^{r}(g)=g y, y \in G_{n-1}$ and $\phi^{r}(y)=y y^{\prime}, y^{\prime} \in G_{n-2}$, it follows by a direct computation that

$$
[g]_{r}^{j}=y_{j} y^{j(j-1) / 2} g^{j}, \quad \text { where } y_{j} \in G_{n-2} .
$$

From (39) it follows that the least value of $j$ for which $[g]_{r}^{j}$ is in $G_{n-1}$ is $j=p$, and that $G_{n-1}$ is of index $r p$ with respect to the generator $[g]_{r}^{p}$. Since

$$
\left[x^{-1} \phi^{\tau}(x)\right]_{r}^{p}=x^{-1} \phi^{\tau p}(x),
$$


$x \in G_{i}$ implies

$$
x^{-1} \phi^{\tau p}(x) \in G_{i-1} .
$$

Hence we may apply induction to $G_{n-1}$ to conclude that the automorphism

$$
\phi_{1}=\phi^{p^{n-2}\left(p^{m}-1\right)}
$$

leaves $G_{n-1}$ elementwise fixed.

But then

$$
\left(\phi_{1}(g)\right)^{p}=\phi_{1}\left(g^{p}\right)=g^{p},
$$

whence $\phi_{1}(g)=g z$, with $z^{p}=1$. But then $z \in G_{n-1}, \phi_{1}(z)=z$ and $\phi_{1}{ }^{p}(g)=g$. It follows that

$$
\phi_{1}^{p}=\phi^{p^{n-1}\left(p^{m}-1\right)}
$$

is the identity on $G$.

For the second part of the lemma we need the statement:

$$
[g]_{\tau}^{j} \in G_{n-1} \quad \text { if and only if } p^{i} \mid j .
$$

We have proved (40) above for $i=1$. If $i>1$, set $g_{1}=[g]_{r}{ }^{p}$. Since $g_{1}$ is a generator of $G_{n-1}$ of index $r p$, it follows by induction that

$$
\left[g_{1}\right]_{r p}^{k} \in G_{n-i}
$$

if and only if $p^{i-1} \mid k$. But now by Lemma 2.1 ,

$$
\left[g_{1}\right]_{r p}^{k}=[g]_{r}^{p k},
$$

and (40) follows at once.

In particular, (40) implies that

$$
[g]_{r}^{p^{n}}=1
$$

and that there are exactly $p^{n}-p^{n-1}$ values of $j<p^{n}$ for which $[g]_{r}^{j}$ has order $p^{n}$. For these values of $j$ the elements $\phi^{i}\left([g]_{r}^{j}\right)$ must exhaust the $p^{m n}-p^{m(n-1)}$ elements of $G$ of order $p^{n}$. Hence

$$
h\left(p^{n}-p^{n-1}\right) \geqslant p^{m n}-p^{m(n-1)} \text {. }
$$

But $h \mid p^{n-1}\left(p^{m}-1\right)$, whence

$$
p^{n-1}\left(p^{m}-1\right)\left(p^{n}-p^{n-1}\right) \geqslant p^{m n}-p^{m(n-1)} \text {. }
$$

It follows that $p-1 \geqslant p^{m(n-1)-2 n+2}$, and we conclude from this inequality that either $n=1$ or $m \leqslant 2$.

The following theorem will be of considerable importance in determining the structure of a regular $\phi$-group.

THEOREM 6. Assume that a regular $\phi$-group $G$ of index $r$ and order $p^{a}$ contains a sequence of normal subgroups $G=G_{n} \supset G_{n-1} \supset \ldots \supset G_{1} \supset G_{0}=1$, invariant under $\phi$, such that no subgroup of $G$ invariant under $\phi$ lies properly between $G_{i}$ and $G_{i-1}$ and such that if $x \in G_{i}, x^{-1} \phi^{\tau}(x) \in G_{i-1}, i=1,2, \ldots, n$. Then $G$ is Abelian. 
Proof. We shall first show that all the elements of order $p$ in $G$ are contained in $G_{1}$, and hence that $G_{1}$ lies in the centre of $G . \bar{G}=G / G_{1}$ satisfies all the conditions of the lemma, and hence by induction the elements of order $p$ in $\bar{G}$ are contained in the subgroup $\bar{G}_{2}=G_{2} / G_{1}$, which is Abelian of type (p, p, $\ldots, \mathrm{p})$. Hence the elements of order $p$ in $G$ are contained in $G_{2}$. Since $G_{1}$ is a minimal subgroup of $G$ invariant under $\phi$, it is also Abelian of type (p, p, $\ldots, p)$.

We have $G_{2}$ of index $r s$ with respect to some generator $g_{2}, g_{2}{ }^{p} \in G_{1}$, and $\phi^{r}\left(g_{2}\right)=y g_{2}$ for some $y$ in $G_{1}$. Since $G_{1}$ is normal in $G_{2}$, it follows directly that

$$
\left[g_{2}\right]_{r s}^{j}=z_{j} g_{2}^{j}, \quad z_{j} \text { in } G_{1} .
$$

If $g_{2}$ has order $p^{2}$, we conclude at once from this relation that the elements of order $p$ in $G_{2}$ are contained in $G_{1}$.

On the other hand, suppose $g_{2}{ }^{p}=1$. First of all, if $G_{1}$ were not in the centre $C$ of $G_{2}$, we would have $G_{1} \cap C=1$ and $G_{1} C / G_{1} \cong \bar{G}_{2}$, since no proper subgroup of $\bar{G}_{2}$ is invariant under $\phi$. But then $G_{2}=G_{1} C$, and so $G_{2}$ is Abelian. $G_{1}$ must therefore lie in the centre of $G_{2}$. But now if $\phi^{r s}\left(g_{2}\right)=z g_{2}, z \in G_{1}$, it follows that

$$
\left[g_{2}\right]_{r s}^{j}=z^{j(j-1) / 2} g_{2}^{j}
$$

If $p$ is odd, we conclude that $\left[g_{2}\right]_{r s}{ }^{p}=g_{2}{ }^{p}=1$, a contradiction to the fact that $G_{1}$ is spanned by the elements of the form

$$
\phi^{i}\left([g]_{r s}^{p j}\right) .
$$

If $p=2,(42)$ gives $\left[g_{2}\right]_{r s}{ }^{4}=1$, and so the orbits of the four elements $\left[g_{2}\right]_{r s}{ }^{j}$, $j=1,2,3,4$ must $\operatorname{span} G_{2}$. But

$$
\left[g_{2}\right]_{r s}^{3}=z g_{2}=\phi^{r s}\left(g_{2}\right)
$$

since $g_{2}{ }^{2}=1$, and hence $\left[g_{2}\right]_{r s}{ }^{1}$ and $\left[g_{2}\right]_{r s}{ }^{3}$ determine the same orbit. It follows that the orbit of $g_{2}$ under $\phi$ must include every element of $G_{2}-G_{1}$, whence

$$
h \geqslant o\left(G_{2}\right)-o\left(G_{1}\right) .
$$

Since our assumptions imply that every element of $G_{2}$ is of order $2, G_{2}$ is Albelian and we may regard $\phi$ as a linear transformation of an $n$-dimensional vector space $\left(2^{n}=o\left(G_{2}\right)\right)$, over the field with 2 elements, which leaves some $t$-dimensional subspace invariant $\left(2^{t}=o\left(G_{1}\right)\right)$. But the maximum order of such a linear transformation is easily computed to be $\left(2^{t}-1\right)\left(2^{n-t}-1\right)$, which is less than $2^{n}-2^{t}$, in contradiction to (43). Hence $G_{1}$ consists of the elements of order dividing $p$ in $G$, as asserted.

If $o\left(G_{1}\right)=p, G$ therefore has a unique subgroup of order $p$, and as is well-known, this implies that $G$ is either cyclic or isomorphic to the generalized quaternion group of order $2^{a}$. But this last group has a unique element of order 2, which is necessarily fixed by every automorphism of the group. 
Hence $G$ is Abelian if $G_{1}$ is cyclic. We assume therefore that $o\left(G_{1}\right)=p^{t}$ with $t \geqslant 2$.

We consider the group $\bar{G}=G / G_{1}$, and suppose $k$ to be the order of the image $\bar{\phi}$ of $\phi$ on $\bar{G}$. If $F$ denotes the subgroup of $G$ left elementwise fixed by $\phi^{k}$, we have $F \cap G_{1}=1$ or $F \cap G_{1}=G_{1}$, since $G_{1}$ is a minimal subgroup of $G$ invariant under $\phi$ and since $F$ is also invariant under $\phi$. Since $G_{1}$ contains every element of order $p$ in $G, F \cap G_{1}=1$ implies $F=1$.

Consider the case $F=1$. If $g$ is a generator of $G, \phi^{k}(g)=z_{1} g, z_{1}$ in $G_{1}$; and since $\phi^{k}$ leaves only the identity element of $G_{1}$ fixed, $z_{1}=x^{-1} \phi^{k}(x)$ for some $x$ in $G_{1}$, and $\phi^{k}\left(x^{-1} g\right)=x^{-1} g$. Thus $x^{-1} g \in F$, whence $g=x$. Thus $G=G_{1}$ is Abelian. We may thus suppose $F \supset G_{1}$.

Now $\bar{G}$ satisfies all the hypotheses of the theorem and is Abelian by induction. But then it satisfies the conditions of Lemma 6.1, and consequently is either cyclic, of type $\left(p^{n-1}, p^{n-1}\right)$ or of type $(p, p, \ldots, p)$ and order $p^{m}$ with $k \mid p^{m}-1$. If $\bar{G}$ is cyclic, $G$ is of course Abelian, since $G_{1}$ is in its centre. In the second case, it follows that every element of $G$ is of the form $x g^{i} \phi(g)^{j}$ for some element $x$ in $G_{1}$ and suitable integers $i, j$. Suppose now that

$$
\phi(g) g=y g \phi(g),
$$

$y$ in $G_{1}$.

Since $\bar{G}$ is of type $\left(p^{n-1}, p^{n-1}\right), \bar{\phi}^{2}(\bar{g})=\bar{g}^{\alpha} \bar{\phi}\left(\bar{g}^{\beta}\right)$ for some integers $\alpha, \beta$, where $\bar{g}$ denotes the image of $g$ in $\bar{G}$. Hence

$$
\phi^{2}(g)=z g^{\alpha} \phi\left(g^{\beta}\right)
$$$$
z \in G_{1} \text {. }
$$

Now apply $\phi$ to (44) and use (45) to obtain

$$
\begin{aligned}
\phi^{2}(g) \phi(g)=\phi(y) \phi(g) & \phi^{2}(g)=\phi(y) \phi(g) z g^{\alpha} \phi\left(g^{\beta}\right) \\
& =\phi(y) y^{\alpha}\left(z g^{\alpha} \phi\left(g^{\beta}\right)\right) \phi(g)=\phi(y) y^{\alpha} \phi^{2}(g) \phi(g) .
\end{aligned}
$$

Hence $\phi(y)=y^{-\alpha}$, and the subgroup $H$ generated by $y$ is invariant under $\phi$. Since $H \subset G_{1}$, we have $H=G_{1}$ or $H=1$. In the first case, $o\left(G_{1}\right)=p$, contrary to our present assumption. Hence $y=1$ and it follows at once from (44) that $G$ is Abelian.

There remains the case $F \supset G_{1}, \bar{G}$ Abelian of type $(p, p, \ldots, p)$ and order $p^{m}$, with $k \mid p^{m}-1$. In this case the relation $\phi^{k}(g)=z_{1} g$ implies $\phi^{k p}(g)=g$, whence

$$
h|k p|\left(p^{m}-1\right) p .
$$

On the other hand, as in the proof of Lemma 10.1

$$
[g]_{r}^{p^{2}}=1
$$

and hence

$$
h p^{2}>o(G)=o\left(G_{1}\right) o(\bar{G})=p^{t+m} .
$$

Combining (22) and (23), we get the inequality $\left(p^{m}-1\right) p^{3}>p^{t+m}$, which implies $t \leqslant 2$. We are assuming $t>1$ and hence $t=2$. 
The theorem has already been proved if $m \leqslant 2$. Hence we may assume $m \geqslant 3$. Let $\bar{y}_{1}, \bar{y}_{2}, \ldots, \bar{y}_{m}$ be a basis for $\bar{G}$ and $y_{1}, y_{2}, \ldots, y_{m}$ a set of representatives in $G$. Since $G_{1}$ contains all elements of $G$ of order $p, y_{i}{ }^{p} \neq 1$ for all $i$. Since $y_{i}{ }^{p} \in G_{1}$ and $G_{1}$ is of type $(p, p)$, there exists integers $\gamma_{1}$ and $\gamma_{2}$ such that

$$
y_{3}^{p}=y_{1}^{p \gamma_{1}} y_{2}^{p \gamma_{2}}
$$

On the other hand, if

$$
y_{3}\left(y_{1}^{\gamma_{1}} y_{2}^{\gamma_{2}}\right)=x_{1} y_{1}^{\gamma_{1}} y_{2}^{\gamma_{2}} y_{3} \quad \text { and } \quad y_{2}^{\gamma_{2}} y_{1}^{\gamma_{1}}=x_{2} y_{1}^{\gamma_{1}} y_{2}^{\gamma_{2}}
$$

then

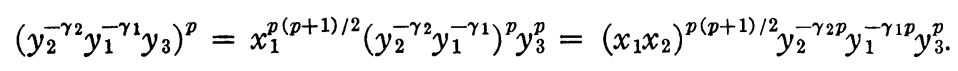

If $p$ is odd, it follows at once from (49) and (50) that $y_{2}^{-\gamma_{2}} y_{1}-\gamma_{1} y_{3}$ has order $p$ and hence is in $G_{1}$. We conclude that $\bar{y}_{3}=\bar{y}_{1}{ }^{\gamma_{1}} \bar{y}_{2}^{\gamma_{2}}$, which implies $m \leqslant 2$, a contradiction.

On the other hand, if $p=2$, and $\bar{g}$ is the residue of $g$ in $\bar{G}$, it follows as in the first part of the proof that $[g]_{r}{ }^{4}=1$, that $[g]_{r}{ }^{3}$ and $[g]_{r}{ }^{1}$ determine the same orbits, and hence that the orbit of $g$ under $\phi$ must include all $2^{2}\left(2^{m}-1\right)$ elements of $G-G_{1}$, and hence

$$
h \geqslant 2^{2}\left(2^{m}-1\right) \text {. }
$$

On the other hand, since every element of $G_{1}$ is of the form $\phi^{i}\left(g_{1}^{j}\right), \phi$ has order 3 on $G_{1}$. Since $F \supset G_{1}, 3 \mid k$. But then $\phi^{k}(g)=x g, x \in G_{1}$, implies $\phi^{2 k}(g)=g$, whence $h \mid 2 k$. Since $k \leqslant 2^{m}-1, h \leqslant 2\left(2^{m}-1\right)$ in contradiction to $(51)$.

Conollary. A regular $\phi$-group of index 0 and of prime power order is Abelian.

The structure of $\phi$-groups of index 0 is now easily obtained.

Theorem 7. A regular $\phi$-group of index 0 is Abelian.

Proof. If $G$ is of index 0 , so is every one of its subgroups. Since $\phi$ is regular, $\phi$ leaves some $p$-Sylow subgroup of $G$ invariant for every $p \mid o(G)$. It follows from the preceding corollary that the Sylow subgroups of $G$ are all Abelian. and hence by the corollary of Theorem 1, that $G$ is Abelian.

\section{The structure of regular $\phi$-groups of prime power order.}

THEOREM 8. A regular $\phi$-group of prime power order is either Abelian or metabelian.

Proof. Let $G$ be a regular $\phi$-group of index $r$ and order $p$. We shall first prove that $G$ contains a normal subgroup $F^{*}$ invariant under $\phi$ and of index $r s$ such that 
(a) $F^{*}$ satisfies the hypotheses of Theorem 6 .

(b) $\widetilde{G}=G / F^{*}$ is Abelian of type $(p, p, \ldots, p)$.

(c) The image $\tilde{\phi}^{r}$ of $\phi^{r}$ leaves only the identity element of $\widetilde{G}$ fixed.

(d) If $k=$ order of $\tilde{\phi}$, then $(k, p)=1$ and $k \mid r s$.

We shall then show that $F^{*}$ is actually in the centre of $G$.

Suppose first that $\phi^{r}$ leaves some proper subgroup $F$ of $G$ elementwise fixed. By Theorem 2, F is normal in $G$. Let $\bar{G}=G / F$. By induction $\bar{G}$ contains a subgroup $\bar{F}^{*}$ of index $r s$ such that $\bar{F}^{*}, \widetilde{G}=\bar{G} / \bar{F}^{*}$, and the image $\tilde{\phi}$ of $\phi$ on $\widetilde{G}$ satisfy conditions (a) to (d). If $F^{*}$ denotes the inverse image of $\bar{F}^{*}$ in $G$, $F^{*}$ is of index $r s$. Since $F$ is of index 0 , it follows readily from Lemma 6.1 and condition (a) for $\bar{F}^{*}$ that $F^{*}$ satisfies (a). Since $G / F^{*} \cong \widetilde{G}$, the remaining conditions follow at once.

We may therefore assume that $\phi^{r}$ leaves only the identity element of $G$ fixed. If $G$ is Abelian of type $(p, p, \ldots, p)$ and $(h, p)=1$, where $h=$ order of $\phi$, set $F^{*}=1$.

If $G$ is not of this form, let $C_{1}$ be a minimal subgroup of the centre of $G$, invariant under $\phi$, and set $\bar{G}=G / C_{1}, \bar{\phi}=$ image of $\phi$ on $\bar{G}$. If $\bar{G}$ is Abelian of type $(p, p, \ldots, p)$ and the order $m$ of $\bar{\phi}$ is relatively prime to $p$, we let $H$ be the subgroup of elements of $G$ left elementwise fixed by $\phi^{m}$. If $H \cap C_{1}=1$, it follows by the usual argument that $G=C_{1} H$, that $H \cong \bar{G}$, and consequently that $G$ is Abelian of type $(p, p, \ldots, p)$. Since $C_{1}$ is a minimal subgroup of $G$ invariant under $\phi$, the order of $\phi$ on $C_{1}$ is relatively prime to $p$, and it follows at once that $(h, p)=1$, a contradiction. Thus $H \supset C_{1}$.

Let $g$ be a generator of $G$ of index $r$ and $g_{1}=[g]_{r}^{s}$ a generator of $C_{1}$ of index $r s$. If $\bar{g}$ is the residue of $g$ in $\bar{G}$, $[\bar{g}]_{r}{ }^{s}=1$, and since $\bar{\phi}$ leaves only the identity element of $\bar{G}$ fixed, it follows that $\bar{\phi}^{r s}(\bar{g})=\bar{g}$. Thus $m \mid r s$. Since $H \supset C_{1}$ we conclude that $x^{-1} \phi^{r s}(x)=1$ for all $x$ in $C_{1}$. If we put $F^{*}=C_{1}$, it is clear that conditions (a) to (d) hold.

Consider then the case in which either $\bar{G}$ is not Abelian of type $(p, p, \ldots, p)$ or $(m, p) \neq 1$ so that $\bar{G}$ contains at least one proper normal subgroup invariant under $\phi$. By induction $\bar{G}$ contains a proper normal subgroup $\bar{F}^{*}$ of index $r s$ such that if $\widetilde{G}=\bar{G} / \bar{F}^{*}, \tilde{\phi}=$ image of $\bar{\phi}$ on $\widetilde{G}$, and $k=\operatorname{order}$ of $\tilde{\phi}$, then $\bar{F}^{*}$ satisfies the conditions of Theorem $6, \widetilde{G}$ is Abelian of type $(p, p, \ldots, p)$, $(k, p)=1$ and $k \mid r s$, and $\tilde{\phi}^{r}$ is Frobenius. Our conditions imply that $\bar{\phi}^{r s}(\bar{g})=\bar{x} \bar{g}$, $\bar{x} \in \bar{F}^{*}$. It follows as in the derivation of (39) and (40) that

$$
\bar{\phi}^{r s p^{n}}(\bar{g})=\bar{g}
$$

for some integer $n$, and hence

$$
m \mid r s p^{n}
$$

Let $H$ be the subgroup of $G$ left elementwise fixed by $\phi^{r s p^{n}}$, and suppose first that $H \supset C_{1}$. Let $F^{*}$ be the inverse image of $F^{*}$ in $G$. The index of $F^{*}$ $=$ index of $\bar{F}^{*}=r s$. Furthermore $\phi^{r s p^{n}}(x)=x$ for all $x$ in $C_{1}$. Since $C_{1}$ is a minimal subgroup of $G$ invariant under $\phi$, the order of $\phi$ on $C_{1}$ is relatively 
prime to $p$, and hence $x^{-1} \phi^{r s}(x)=1$ for all $x$ in $C_{1}$. It follows immediately that $F^{*}$ satisfies (a). Since $G / F^{*} \cong \bar{G} / \bar{F}^{*}$, (b), (c), and (d) also hold.

On the other hand, if $H \cap C_{1}=1$, it follows once again that $G=C_{1} H$ and that $\bar{G} \cong H$ under an isomorphism $\tau$ such that $(\tau \bar{\phi}(\bar{x}))=\phi(\tau(\bar{x}))$ for all $\bar{x}$ in $\bar{G}$. Let $F^{\prime}$ be the normal subgroup of $H$ which corresponds to $\bar{F}^{*}$ under $\tau$. Then $F^{\prime}$ is invariant under $\phi, \phi$ has order $m$ on $H$ and $m \mid r s p$. Let $F_{1}$ be a minimal subgroup of $F^{\prime}$ invariant under $\phi$. Since every subgroup of $F^{\prime}$ invariant under $\phi$ is characteristic in $F^{\prime}, F_{1}$ is normal in $H$ and hence also in $G$. Let $G^{\prime}=G / F_{1}, \phi^{\prime}=$ image of $\phi$ on $G^{\prime}, m^{\prime}=$ order of $\phi^{\prime}$. By induction $G^{\prime}$ contains a normal subgroup $F^{\prime *}$ of index $r s^{\prime}$ such that conditions (a), (b), (c) hold for $F^{\prime *}$ and $\widetilde{G}=G / F^{\prime *}$. In particular, $m^{\prime}=r s^{\prime} p^{n \prime}$ for some integer $n^{\prime}$. Let $H_{1}$ be the subgroup of $G$ invariant under

$$
\phi^{r s^{\prime} p^{n}} \text {. }
$$

Since $\bar{F}^{*}$ is the homomorphic image of $C_{1} F^{\prime}, C_{1} F^{\prime}$ is of index $r s$. Since $F_{1} \subset C_{1} F^{\prime}$, it follows that $r s \mid r s^{\prime}$, and hence $H_{1} \supset F_{1}$. Our desired conclusion now follows as in the preceding paragraph.

It remains to prove that $F^{*}$ lies in the centre of $G$. By construction $F^{*}$ contains a sequence of normal subgroups $F^{*}=F_{n} \supset F_{n-1} \supset \ldots \supset F_{1} \supset F_{0}=1$ invariant under $\phi$ such that

$$
x^{-1} \phi^{r s}(x) \in F_{i-1} \text { if } x \in F_{i}
$$

and such that no proper subgroup of $F^{*}$ invariant under $\phi$ lies properly between $F_{i}$ and $F_{i-1}$. By Theorem $6, F^{*}$ is Abelian. It is easy to see that this implies that $F^{*}$ is of type $\left(p^{n}, p^{n}, \ldots, p^{n}\right)$ and that $F_{i}$ is the subgroup generated by the elements of order $p^{i}$ in $F^{*}$. Thus $F_{1}$ is characteristic in $F^{*}$, and consequently normal in $G$. Since $F^{*}$ is a minimal subgroup of $G$ invariant under $\phi$, we conclude that $F_{1}$ lies in the centre of $G$.

Since $\widetilde{G}$ is Abelian of type $(p, p, \ldots, p)$ we can decompose $\widetilde{G}$ into the direct product of subgroups $\widetilde{G}_{j}, j=1,2, \ldots, t$ invariant under $\tilde{\phi}^{r s}$ and none of which can be further decomposed into proper subgroups invariant under $\tilde{\phi}^{r s}$. If $G_{j}$ denotes the inverse image of $\widetilde{G}_{j}$, it suffices to prove that $F^{*}$ lies in the centre of each $G_{j}$. For definiteness, take $j=1$.

First of all, if $\tilde{\phi}^{r s}$ has non-trivial fixed elements on $\widetilde{G}_{1}$, it follows from the minimality of $\widetilde{G}_{1}$ that $\tilde{\phi}^{r s}$ is in fact the identity on $\widetilde{G}_{1}$. Hence if $x \in G_{1}$, $x^{-1} \phi^{r s}(x) \in F^{*}$. It follows at once that $G_{1}$ is a group of index $r s$ satisfying the conditions of Theorem 6 , and hence is Abelian. Thus $F^{*}$ is in the centre of $G_{1}$ in this case.

Consider then the case in which $\tilde{\phi}^{\text {rs }}$ leaves only the identity element of $\widetilde{G}_{1}$ fixed. $\widetilde{G}_{1}$ has a basis $\tilde{y}_{1}, \ldots, \tilde{y}_{q}$ such that

$$
\tilde{\phi}^{r s}\left(\tilde{y}_{i}\right)=y_{i+1}, \quad i=1,2, \ldots, q-1
$$

and

$$
\tilde{\phi}^{r s}\left(y_{q}\right)=\tilde{y}_{1}^{\alpha_{1}} \tilde{y}_{2}^{\alpha_{2}} \ldots \tilde{y}_{q}^{\alpha q}
$$

for suitable integers $\alpha_{1}, \alpha_{2}, \ldots, \alpha_{q}$. 
If

$$
\tilde{y}_{1}^{i_{1}} \tilde{y}_{2}^{i_{2}} \ldots \tilde{y}_{q}^{i_{q}}
$$

is a fixed element of $\tilde{\phi}^{r s}$, then it is easily checked that the integers $i_{1}, i_{2}, \ldots, i_{q}$ are a solution of the congruences.

$$
\alpha_{1} i_{q} \equiv i_{1} ; \alpha_{2} i_{q}+i_{1} \equiv i_{2} ; \ldots ; \alpha_{q} i_{q}+i_{q-1} \equiv i_{q} \quad(\bmod p),
$$

and conversely. It follows readily from (54) that $\tilde{\phi}^{r s}$ is Frobenius on $\widetilde{G}_{1}$ if and only if

$$
\left(\alpha_{1}+\alpha_{2}+\ldots+\alpha_{q}-1, p\right)=1 .
$$

Let $y_{i}$ be a representative of $\tilde{y}_{i}$ in $G_{1}$ such that

$$
\phi^{r s}\left(y_{i}\right)=y_{i+1}, \quad i=1,2, \ldots, q-1 .
$$

Then

$$
\phi^{r s}\left(y_{q}\right)=x_{0} y_{1}^{\alpha_{1}} y_{2}^{a_{2}} \ldots y_{q}^{a_{q}},
$$

$x_{0} \in F^{*}$. If $\psi$ denotes the automorphism of $F^{*}$ induced by conjugation by $y_{1}$, $\psi$ leaves $F_{1}$ elementwise fixed since $F_{1}$ lies in the centre of $G_{1}$. We shall prove by induction on $n$ that $\psi$ leaves $F^{*}$ elementwise fixed. This will suffice to prove that $F^{*}$ is in the centre of $G_{1}$, and will complete the proof of the theorem.

By induction $F^{*} / F_{1}$ lies in the centre of $G / F_{1}$, whence

$$
\text { if } x \in F^{*}, \quad \psi(x)=z x, \quad z \in F_{1} .
$$

Suppose $\psi$ is the identity on $F_{k}$ with $1 \leqslant k<\mathrm{n}$. We shall prove $\psi$ is the identity on $F_{k+1}$. Applying $\phi^{r s i}$ to (56), we obtain

$$
\phi^{r s i}(\psi(x))=\phi^{r s i}\left(y_{1}\right) \phi^{r s i}(x) \phi^{r s i}\left(y_{1}^{-1}\right)=\phi^{r s i}(z) \phi^{r s i}(x)=z \phi^{r s i}(x) .
$$

But if $x \in F_{k+1}, \phi^{r s i}(x)=x z_{i}, z_{i} \in F_{k}$. Since $F_{k}$ is in the centre of $G_{1}$, we conclude from (57) that

$$
\phi^{r s i}(\psi(x))=z x=\psi(x) \text { for all } i \text { and all } x \text { in } F_{k+1} .
$$

By repeated use of (58) we now obtain

$\psi(x)=\phi^{r s q}(\psi(x))=\left(x_{0} y_{1}^{\alpha_{1}} y_{2}^{\alpha_{2}} \ldots y_{q}^{\alpha_{q}}\right)(x)\left(x_{0} y_{1}^{\alpha_{1}} y_{2}^{\alpha_{2}} \ldots y_{q}^{\alpha_{q}}\right)^{-1}=\psi^{\alpha_{1}+\alpha_{2}+\ldots+\alpha_{q}}(x)$, whence

$$
\psi^{\alpha_{1}+\alpha_{2}+\cdots+\alpha_{q}-1}(x)=x, \quad x \in F_{k+1} .
$$

On the other hand, (56) implies $\psi^{p}(x)=x$. But then (55) and (59) together imply $\psi(x)=x$ for all $k$ in $F_{k+1}$. Q.E.D.

Theorem 8 and Theorem 4 together imply

Theorem 9. A regular $\phi$-group is either Abelian or nilpotent of class 2 .

We conjecture that a regular $\phi$-group is Abelian if $\phi^{r}$ is Frobenius. This result would follow easily from the following conjecture concerning fixed-point free automorphisms of $p$-groups. 
CONJECTURE. Let $G$ be a non-Abelian p-group which admits an automorphism $\phi$ of order $h$ leaving only the identity element of $G$ fixed, and assume that $G$ cannot be expressed as the direct product of two proper subgroups invariant under $\phi$. Then $h^{2}<o(G)$.

12. The relation between $\phi$-groups and groups of the form ABA.

In the proof of the preceding theorem we have already seen that a $\phi$-group $G$ can be imbedded as a normal subgroup of an $A B A$-group $G^{*}$ satisfying $G^{*}=G A$ and $G \cap A=1$. The converse is also true, and consequently we have

THEOREM 10. $G$ is a $\phi$-group if and only if it can be imbedded as a normal subgroup of a group $G^{*}$ of the form $A B A$, where $A$ and $B$ are cyclic subgroups of $G^{*}$, such that $G^{*}=G A$ and $A \cap G=1$.

Proof. It suffices to prove that if an $A B A$-group $G^{*}$ in which $A, B$ are cyclic contains a normal subgroup $G$ such that $G^{*}=G A$ and $G \cap A=1$, then $G$ is a $\phi$-group.

If $a, b$ are generators of $A, B$ respectively, we have $b=g a^{r}$ for some element $g$ in $G$ and some integer $r$. Since $G$ is normal, the elements

$$
\begin{aligned}
& b^{j} a^{-j r}=\left(b a^{-r}\right)\left(a^{r} b a^{-2 r}\right) \ldots\left(a^{(j-1) r} b a^{-j r}\right)=g\left(a^{r} g a^{-r}\right) \ldots \\
& a^{(j-1) \tau} g a^{-(j-1) r}
\end{aligned}
$$

are in $G$ for ali $i, j$.

Suppose for some $j, b^{j} a^{k} \in G$; then $a^{-k-j r}=\left(b^{j} a^{k}\right)^{-1}\left(b^{j} a^{-j r}\right) \in G \cap A$. Since $G \cap A=1, a^{k}=a^{-j r}$. It follows at once that $G$ consists precisely of the elements of $G^{*}$ of the form $a^{i} b^{j} a^{-j r-i}$. If we now define $\phi$ to be an automorphism of $G$ induced by conjugation by $a$, it follows as in the proof of Theorem 5 that every element of $G$ is of the form $\phi^{i}\left([g]_{r}^{j}\right)$. Thus $G$ is a $\phi$-group of index $r$ and with generator $g$.

Combining Theorems 5 and 10, we obtain our final result:

Theorem 11. A group $G^{*}$ which is of the form $A B A$, where $A$ and $B$ are cyclic subgroups, and which contains a normal subgroup $G$ such that $G^{*}=G A$ and $G \cap A=1$ is solvable.

In a subsequent paper we shall show that an $A B A$ group $G^{*}$ with a trivial centre in which $A$ is its own normalizer and $A$ is of odd order always contains a normal subgroup $G$ such that $G^{*}=G A$ and $G \cap A=1$. We shall also determine the structure of $G^{*}$ when $o(A)$ is even and, in particular, shall show that $G^{*}$ is solvable. 


\section{REFERENCES}

1. W. Burnside, Theory of groups of finite order (Dover, New York, 1955).

2. W. Feit, On the structure of Frobenius groups, Can. J. Math., 9 (1957), 587-96.

3. D. Gorenstein, A class of Frobenius groups, Can. J. Math., 11 (1959), 39-42.

4. D. Gorenstein and I. N. Herstein, A class of solvable groups, Can. J. Math., 11 (1959), 311-20.

5. G. Higman, Groups and rings which have automorphisms without non-trivial fixed elements, J. London Math. Soc., 32 (1957), 321-334.

6. H. Zassenhaus, The theory of groups (Chelsea, New York, 1958).

Clark University 